Review Article

\title{
Thermal Transport across Solid Interfaces with Nanoscale Imperfections: Effects of Roughness, Disorder, Dislocations, and Bonding on Thermal Boundary Conductance
}

\author{
Patrick E. Hopkins \\ Department of Mechanical and Aerospace Engineering, University of Virginia, Charlottesville, VA 22904, USA \\ Correspondence should be addressed to Patrick E. Hopkins; phopkins@virginia.edu
}

Received 20 November 2012; Accepted 7 December 2012

Academic Editors: Y. He, T. Ohara, and Z. Yu

Copyright (C) 2013 Patrick E. Hopkins. This is an open access article distributed under the Creative Commons Attribution License, which permits unrestricted use, distribution, and reproduction in any medium, provided the original work is properly cited.

\begin{abstract}
The efficiency in modern technologies and green energy solutions has boiled down to a thermal engineering problem on the nanoscale. Due to the magnitudes of the thermal mean free paths approaching or overpassing typical length scales in nanomaterials (i.e., materials with length scales less than one micrometer), the thermal transport across interfaces can dictate the overall thermal resistance in nanosystems. However, the fundamental mechanisms driving these electron and phonon interactions at nanoscale interfaces are difficult to predict and control since the thermal boundary conductance across interfaces is intimately related to the characteristics of the interface (structure, bonding, geometry, etc.) in addition to the fundamental atomistic properties of the materials comprising the interface itself. In this paper, I review the recent experimental progress in understanding the interplay between interfacial properties on the atomic scale and thermal transport across solid interfaces. I focus this discussion specifically on the role of interfacial nanoscale "imperfections," such as surface roughness, compositional disorder, atomic dislocations, or interfacial bonding. Each type of interfacial imperfection leads to different scattering mechanisms that can be used to control the thermal boundary conductance. This offers a unique avenue for controlling scattering and thermal transport in nanotechnology.
\end{abstract}

\section{Introduction}

As the world's population and power demands increase, our technological solutions continue to rely on power-hungry applications. The shear population increase in the world along with skyrocketing electricity, power, and transportation needs in emerging markets has led to necessary minimum levels of energy for sustainable growth and livelihood of human kind. The trends in energy use are marked by our society's continued advancement of technology, communication, transportation, and quality of life. For example, in 2010, the United States used an estimated 28.7 trillion $\mathrm{kW}$-h of energy [1]. These massive energy demands both in the USA and abroad are a necessary requirement for continued capabilities and qualities of life that have been made possible with the advancement of technologies. For example, increased computing needs (internet, cloud computing, and wireless communications), although more energy efficient in the recent past, have led to an increase in data center energy usage by roughly a factor of six over the past decade [2]; although technologies have become more energy efficient, due to the shear increase in the numbers of users and information, energy consumption and needs are skyrocketing, leading to these astounding levels of energy usage.

The primary fuel for this electricity generation comes from coal, a nonrenewable resource that leads to 2,250 lbs/ MW-h of carbon dioxide, $13 \mathrm{lbs} / \mathrm{MW}-\mathrm{h}$ of sulfer dioxide, and $6 \mathrm{lbs} / \mathrm{MW}-\mathrm{h}$ of nitrogen oxides [2]. This leads to 12.6 billion lbs of $\mathrm{CO}_{2}$ emissions, along with 73 and 34 million lbs of sulfer dioxide and nitrogen oxides, respectively. In addition, more than half (57\%) of the used energy in the USA is rejected as heat where only $43 \%$ of the consumed energy is actually used (work). These stifling statistics create two major foci for energy research: (1) improving the efficiency of the energy usage to reduce the net wasted heat and (2) reducing the harmful emissions of the technologies generating the energy. Improving energy efficiency of devices has been an active area of material research for the last few decades, stemming from 
the drive of the computing industry to maintain Moore's Law [3] and recently focused on mitigating the thermal transport in the nanoscale devices $[4,5]$. Recent novel approaches in reducing the net wasted heat have focused on waste heat recycling via thermoelectric power generation [6-8]. Thermoelectric solutions also show promise in reducing harmful emissions in the grid, as the entirely solid state makeup of thermoelectrics requires no moving parts, cycles, or input fuel other than heat. These thermoelectric solutions combined with "renewable source" technologies (technologies that do not rely on coal as a fuel-such as photovoltaics $[9,10])$ could substantially reduce the carbon footprint of the energy grid.

The issue with these technologies lies in their operation efficiencies, which at this stage of research have boiled down to a thermal engineering problem on the nanoscale. For example, power dissipation limits the performances of electronic systems from individual microprocessors to data centers. In silicon-based microprocessors (by far the most commonly implemented technology), operation beyond a few $\mathrm{GHz}$ is not possible due to on-chip power densities exceeding $100 \mathrm{~W} \mathrm{~cm}^{-2}$, higher than a typical hotplate and far greater than typical cooling capabilities [4]. Even greater power densities have been observed in RF and amplifier devices using III-V or group IV compound semiconductors (e.g., GaAs or $\mathrm{SiC}$ ) [11]. These huge power densities lead to hot-spots and device failure, warranting the need for increased thermal conduction to mitigate the heat load. As another example, current thermoelectric solutions for waste heat recovery are far outperformed by traditional solutions such as heat exchangers with heat pumps or heat engine cycles such as the Organic Rankine or Kalina cycles [6], making major implementations of the more-carbon-footprintfriendly thermoelectric devices impractical. Improvement in the thermoelectric efficiency can be achieved by controlled reduction in thermal transport of the thermoelectric materials, since the thermoelectric efficiency (quantified by the Figure of Merit, ZT) is inversely related to the thermal conductivity. Recent massive improvements in ZT in thermoelectric materials have been discovered by nanostructuring $[8,12-15]$, leaving the research problem to improve energy efficiency centered around understanding thermal transport on the nanoscale. As both increases and decreases in thermal conductivity are required for different applications, the ability to control and tune the thermal properties of materials is therefore the ultimate goal to improve device efficiency and improve energy consumption trends.

This desired thermal control in nanosystems, however, is not a trivial task. Due to the magnitudes of the thermal mean free paths approaching or overpassing typical length scales in nanomaterials (i.e., materials with length scales less than 1 micron), the thermal transport across interfaces can dictate the overall resistance in nanosystems. This leads to a regime in which unique yet relatively unknown mechanisms such as interfacial scattering, ballistic transport, and waveeffects offer potential new degrees of freedom in the thermal engineering of material systems $[5,16]$. However, the fundamental mechanisms driving these electron and phonon

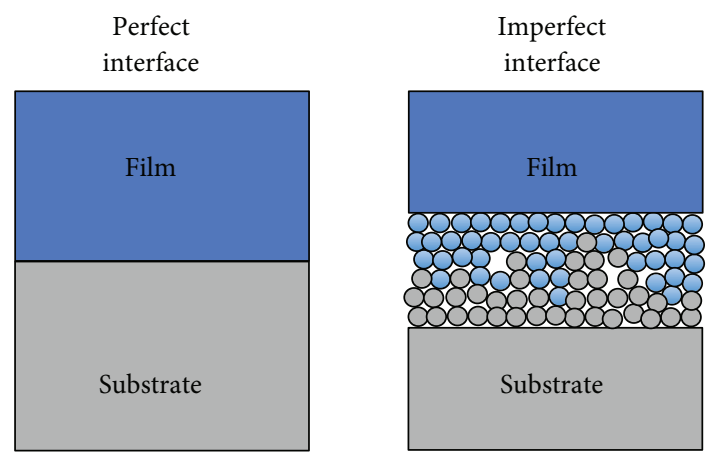

FIgURE 1: Schematic of a perfect and an atomically imperfect interface. A perfect interface is an atomically abrupt, coherent interface between two solids in intimate contact. The atomically imperfect interface depicted here occurs when the interface is not well defined due to atomic disorder (both structural and compositional), resulting in roughness and impurities around the interface.

interactions at nanoscale interfaces are difficult to predict and control since the thermal boundary conductance across interfaces (often called the Kapitza conductance), $h_{\mathrm{K}}[17,18]$, is intimately related to the characteristics of the interface (structure, bonding, geometry, etc.) in addition to the fundamental atomistic properties of the materials comprising the interface itself. This interplay between interfacial properties and thermal boundary conductance can lead to additional carrier scattering mechanisms and phenomena that will not only change the thermal processes, but can also be used to systematically manipulate and control thermal transport in nanosystems.

As an example, consider a "perfect" interface and an atomically disordered, "imperfect" interface, depicted in Figure 1. The imperfect interface introduces roughness along with atomic imperfections that cause additional thermal scattering which can change $h_{\mathrm{K}}$, as I have discussed in previous works [19-24] and will detail throughout this paper. The properties of this interface dictate the additional scattering mechanisms. Therefore, an understanding of the effects of interfacial imperfections and other "non-idealities" (such as roughness, disorder, and bonding) on thermal transport across complex nanoscale interfaces is warranted to fully develop the potential to control electron and phonon thermal processes in nanosystems.

To this end, in this paper I will review recent advancements in the underlying physics of thermal boundary conductance at solid interfaces, focusing specifically on the role of interfacial nanoscale "imperfections," such as surface roughness, compositional disorder, atomic dislocations, or interfacial bonding. In the next section, I present an overview of thermal boundary conductance and discuss pertinent measurements of thermal boundary conductance between two solids to put this thermophysical quantity into perspective. Following this background, I discuss time domain thermoreflectance in Section 3. TDTR has become a common, effective experimental technique to accurately measure the thermal boundary conductance between two solids. In Section 4, 
I will discuss the mathematical semiclassical formalisms for predicting thermal boundary conductance, which are simple, analytical expressions that can be used to understand the electron or phonon transmission across interfaces. Following this, the remainder of the sections will focus on examining the effects of various interfacial imperfections on either the electron- or phonon-dominated thermal boundary conductance across interfaces. These sections will focus on the effects of interfacial interspecies mixing on electron and phonon thermal boundary conductance (Sections 5 and 6, resp.) and roughness, dislocations, and bonding effects on phonon thermal boundary conductance (Sections 7, 8, and 9, resp.).

\section{Background: Thermal Boundary Conductance}

The thermal transport across solid interfaces is quantified by the thermal boundary conductance, $h_{\mathrm{K}}$, or Kapitza conductance [18], which quantifies the efficacy of heat flow across an interface. Mathematically, the thermal boundary conductance is the proportionality constant that relates the heat flux across an interface to the temperature drop associated with that interfacial region via

$$
h_{\mathrm{K}}=\frac{q_{\text {int }}}{\Delta T},
$$

where $q_{\text {int }}$ is the thermal flux across the solid interfacial region and $\Delta T$ is the temperature drop across the interfacial region. It is also often intuitive to conceptualize the inverse of this quantity, the thermal boundary resistance, $R_{\mathrm{K}}=1 / h_{\mathrm{K}}$, which can be paralleled to an electrical resistance network. Note that thermal boundary conductance is discussed here in terms of two materials in intimate contact, thereby making this inherently different than a "contact conductance" which is related to the voids or lack of contact between two solids.

Measurements of the thermal boundary conductance between two solids typical occur in a thin film geometry or at ultralow temperatures. In both of these cases, the resistance due to the interface is a major resistance in the measured system of interest, thereby allowing for accurate measurement sensitivity. One of the first comprehensive reviews of thermal boundary conductance was detailed by Swartz and Pohl in 1989 [17], and their review mainly focused on low temperature measurements and phenomena. Recently, with the advances in sensitive measurement techniques for interrogating thermal transport on the nanoscale [25], accurate measurements of thermal boundary conductance in nanosystems and at elevated temperatures have been achieved $[4,5]$.

As the goal of this paper is to discuss the effects of interfacial "non-idealities" on thermal boundary conductance, only a subset of the measurements of thermal transport across various interfaces will be discussed here. The reader is referred to $[4,5,26]$ for overviews of thermal boundary conductance at "smooth" or seemingly "perfect" interfaces. However, it is informative to discuss the limits of thermal



FIGURE 2: Thermal boundary conductance at various solid interfaces (Pd/Ir [27], Al/Cu [28], TiN/MgO [29], Al/Si [19, 21], Al/single layer graphene (SLG) [24], GaSb/GaAs [23], and Bi/diamond [30]). Typical values span over three orders of magnitude. For comparison, the equivalent conductances of various thicknesses of $\mathrm{SiO}_{2}$ are shown as the solid lines.

boundary conductance at "perfect" interfaces and the difference between electron-dominated and phonon-dominated thermal boundary conductances.

Figure 2 shows the thermal boundary conductance as a function of temperature for various interfaces in which $h_{\mathrm{K}}$ is dominated by either electron or phonon scattering $[21,23,24,27-30]$. The TiN/MgO and $\mathrm{Bi} /$ diamond data represent the highest and lowest phonon-dominated thermal boundary conductance ever measured, respectively [29, 30]. Note that the electron-dominated thermal boundary conductances $(\mathrm{Al} / \mathrm{Cu}$ and $\mathrm{Pd} / \mathrm{Ir})$ are roughly one order of magnitude higher than the phonon-dominated thermal boundary conductances and have a steeper increase with temperature $[27,28]$. Along with these data, the equivalent conductances of various thicknesses of $\mathrm{SiO}_{2}$ are indicated by the solid lines; this equivalent conductance is calculated by $h_{\mathrm{SiO}_{2}}=$ $\kappa_{\mathrm{SiO}_{2}} / d$, where $\kappa_{\mathrm{SiO}_{2}}$ is the thermal conductivity of $\mathrm{SiO}_{2}$ and $d$ is the thickness, as indicated in [31]. Therefore, at room temperature, the thermal boundary conductance between a $\mathrm{Pd} / \mathrm{Ir}$ interface offers the equivalent resistance as roughly $1 \AA$ of $\mathrm{SiO}_{2}$. The highest phonon-dominated thermal boundary conductance offers the same resistance as $\sim 2 \mathrm{~nm}$ of $\mathrm{SiO}_{2}$, nearly 20 times greater resistance than the electron-electrondominated interface. At the lower end of the spectrum, 
the phonon transport across a $\mathrm{Bi}$ /diamond interface is equivalent to the resistance of $\sim 200 \mathrm{~nm}$ of $\mathrm{SiO}_{2}$. Clearly, in nanomaterials and composites, the thermal boundary conductance across solid interfaces can offer a major impediment to heat flow that can dominate the thermal transport in the overall system.

The additional data in Figure 2 ( $\mathrm{Al} / \mathrm{Si}, \mathrm{Al} /$ single layer graphene (SLG), and $\mathrm{GaSb} / \mathrm{GaAs}$ interfaces) represent our recent measurements to examine the effects of some interfacial imperfections on thermal transport across interfaces $[21,23,24]$. The specific interfacial imperfection that was investigated in each material case is indicated by labels in Figure 2. These, along with other measurements, will be discussed in detail throughout this paper. Briefly, these three example interfaces show the effects of either roughness (Al/Si), bonding (Al/SLG), or dislocations ( $\mathrm{GaSb} / \mathrm{GaAs})$ on the thermal boundary conductance. In the $\mathrm{Al} / \mathrm{Si}$ case, a rougher substrate leads to a decrease in thermal boundary conductance [21]. At the Al/SLG interfaces, by increasing the bonding between otherwise nonreacting $\mathrm{Al}$ films and SLG substrates, Al can bond covalently to the SLG, thereby increasing the thermal transport [24]. At the GaSb/GaAs interfaces, the large lattice mismatch between the GaSb and GaAs leads to strain dislocations. The number of dislocations can be controlled via the growth technique, and we observed that making a GaSb/GaAs interface with a higher number of dislocations per unit area results in a lower $h_{\mathrm{K}}$. These effects-roughness, dislocations, and bonding-will be discussed in more detail in Sections 7, 8, and 9, respectively. However, it is apparent in this preliminary overview that the thermal boundary conductance between two solids is not only a function of the materials comprising the interface, but has a strong dependency on the atomic properties of the interface. Therefore, with careful nanoscale engineering of the interfacial properties, the ability to tune the thermal boundary conductance between two solids can be achieved. In the remainder of this paper, I will discuss specific experimental results and accompanying theory on the thermal transport across imperfect interfaces between two solids, including more detail on the specific imperfect interfaces discussed above. However, before delving into the transport physics driving thermal boundary conductance across imperfect interfaces, measurements of $h_{\mathrm{K}}$ with time domain thermoreflectance will be detailed in the following section.

\section{Time Domain Thermoreflectance: An Effective Tool for Measuring Thermal Boundary Conductance between Two Solids}

Over the past few decades, contact-based techniques or experimental platforms based on resistive thermometry apparati have made substantial progress in measuring the thermal transport in nanosystems due to advances in nanofabrication and increasingly sensitive detection schemes [25]. Common resistive thermometry techniques that have been used to determine temperature rises and heat transport properties in nanosystems are steady-state resistance-based techniques [32-37] and the $3 \omega$ technique [31, 38-43]. These methods are based on the fact that the electrical resistivity of a sample or test strip is related to the temperature chang; therefore, by monitoring the change in electrical resistivity of a reference, the change in temperature can be determined. With precise knowledge of geometry of the nanosystem of interest, the thermal conductivity can be determined from the measured temperatures. The advantages of these techniques are that they are direct measurement of the temperature rise and can be directly related to the thermal conductivity of the sample of interest through only a carefully calibrated electrical resistivity reference and precise knowledge of the sample geometry. The disadvantages, however, in addition to the potential uncertainty in sample geometries, are that these techniques require intimate contact between the electrical leads and the sample platform of interest. Inherent in these contacts are both electrical and thermal contact resistances that must be accounted for [44]. In addition to these measurement difficulties, direct measurement of the thermal boundary conductance between two materials of interest is very difficult without the use of multiple samples. For example, if one were to use a steady-state or frequency domain (such as $3 \omega$ ) electrical resistivity technique to measure the thermal properties of a bilayer sample (say, thin film on thin film on substrate), the measured data would represent the total thermal resistance of the sample. Although the individual film properties could be inferred from multilayer thermal modeling [45], a "heat capacity-less" thin interface could not be differentiated from the individual layers. This is not the case with time domain techniques, however.

Time domain techniques for measuring the thermal transport of materials have proven invaluable for monitoring the thermal conductivity and thermal boundary conductance of multilayer structures due to the fact that the thermal effusivity or thermal diffusivity of a material responds differently than the thermal transport across an interfacial region in the time domain. Several time domain techniques have proven quite useful in measurement of these interfacial thermal properties, including laser flash diffusivity [46], transient electrothermal techniques [47], and thermoreflectance techniques $[48,49]$. With recent progress in pulsed laser systems, thermoreflectance techniques employing short pulses have become a robust metrology to accurately determine the thermal transport in nanosystems, as I outline in the following. These thermoreflectance techniques, which rely on the fundamentals of thermoreflectance spectroscopy of materials, can offer ultrafast characterization of the temperature change of materials in the time domain, which alleviates many of the radiative loss, optical effusivity, and contact issues that plague other techniques.

In thermoreflectance spectroscopy, an oscillating perturbation is applied to the sample of interest and the AC component of the reflected light is monitored [50-57]. For example, in a standard pump-probe geometry, the pump is a modulated laser beam or train of laser pulses that is used to optically and thermally excite some sample at frequency, $f$, while the probe is used to interrogate the small changes in reflectivity of the sample caused by the pump excitation at frequency $f$. To back out heat transport characteristics 
using thermoreflectance, the majority of optical perturbation that causes a change in reflectivity must be directly due to a thermal perturbation. For this reason, metallic systems are ideal to transduce the change in reflectivity into the change in temperature due to the large electronic number density around the Fermi level in metals. This resulting thermoreflectivity change in a metal is related to an electron population that is thermalizing or thermalized to a new distribution due to the pump pulse. Hence, the thermoreflectance of the metal, defined as the change in reflectance due to a change in temperature, is given by

$$
\frac{d R}{R\left(T_{0}\right)}=\frac{R_{\text {excited }}-R\left(T_{0}\right)}{R\left(T_{0}\right)},
$$

where $R$ is the reflectivity, $T_{0}$ is the baseline temperature of the sample, and $R_{\text {excited }}$ is the reflectivity of the metal in the state during and after pump pulse absorption. The thermoreflectance of a metal is dictated by its thermoreflectance coefficient, $d R / d T$, which is typically small, on the order of $10^{-6}-10^{-4} \mathrm{~K}^{-1}$ depending on the temperature and metallic band structure at a given wavelength [58-60]. Therefore lock-in techniques are typically used to measure the thermoreflectance of metals in pump-probe geometries. Using ultrashort laser pulses in pump-probe geometries, the time evolution of the thermoreflectance can be measured $[49,61]$.

The time evolution of the thermoreflectance signal is the basis behind time domain thermoreflectance (TDTR). TDTR has been used in several different geometries with various different pulsed lasers, ranging from nanoseconds to femtoseconds [5, 25, 48, 49, 62-67]. These experiments can be separated into two classes: (1) experiments in which the laser pulses are separated far enough in time in which the interrogated sample is relaxed back down to its equilibrium state before the next pulse arrives and (2) experiments in which the pulses are so closely spaced in time that the pulse arrives at the sample surface before full thermal relaxation of the previous pulse. Recently [68], the experiments in the former class have been characterized as "transient thermoreflectance" where experiments utilizing laser systems producing the later class of excitation have been commonly referred to as "time domain thermoreflectance" (TDTR). In TDTR experiments, the buildup of laser pulse energy leads to a steady-state temperature rise in the sample [69] that can provide additional information for thermal analysis. This paper will limit the review of measurement techniques to TDTR, as the majority of the data presented in the later sections were measured with TDTR techniques.

Due to the recent decrease in cost of femtosecond Ti: Sapphire oscillators, most TDTR systems are centered around these high frequency $(\sim 80 \mathrm{MHz})$ laser systems. A typical cost of a $\mathrm{Ti}$ : Sapphire oscillator from a commercial vendor is $\sim \$ 100,000-\$ 200,000$, depending on the output power and pulse width. The TDTR experiment that I have in my lab at University of Virginia is based off of a Spectra Physics Tsunami with $\sim 3 \mathrm{~W}$ output of $90 \mathrm{fs}$ laser pulses centered at $800 \mathrm{~nm}$. This means that $90 \mathrm{fs}$ laser pulses are emanating from the oscillator with about $37.5 \mathrm{~J}$ of energy every $12.5 \mathrm{~ns}$. The pulse energy is split with a variable beam splitter into a pump path and a probe path. The pump path is directed through an electro-optic modulator (EOM) that can modulate the pulse train upwards of $f=25 \mathrm{MHz}$ (I use the electro-optic modulators sold commercially through ConOptics). In my particular setup, I then focus the modulated pump pulses through a bismuth triborate $(\mathrm{BiBO})$ crystal to frequency double the near infrared pulses $(800 \mathrm{~nm}=$ $1.55 \mathrm{eV})$ to visible wavelengths $(400 \mathrm{~nm}=3.11 \mathrm{eV})$. I then direct the pump pulses through an objective to focus the $400 \mathrm{~nm}$ pump pulses onto the sample surface. The modulated pump pulses produce a temperature rise on the sample surface at the frequency $f$ of the EOM.

The pulse energy that is not directed down the pump path after the variable beam splitter is directed down a mechanical delay stage and then focused collinearly with the pump pulses through the objective; these probe pulses are focused on the sample surface in the middle of the pump pulses. I typically make the pump spot size larger than the probe spot size on the sample surface. This reduces error in our measurements due to probe beam drift and movement. This also creates a onedimensional heating event in the sample, cross-plane to the sample surface (note, if the pump beam waist is decreased to the same spot size as the pump, in-plane thermal transport properties can also be measured; however, this is not of interest for this paper since I will only focus on measurements of thermal boundary conductance across a thin interfacial region where heat transfer is nearly entirely cross-plane) [65, 66]. I typically use infinity corrected objectives with anywhere from $5 \times-20 \times$ magnification, which leads to ranges of pump and probe $1 / e^{2}$ radii anywhere from $25-6 \mu \mathrm{m}$ and $6-4 \mu \mathrm{m}$, respectively, depending on the objective. Note that this is very dependent on the particular setup and the spot size of the pump and probe going into the back face of the objective. The reflected probe beam is then reflected along the same incident path, passed through polarizing and color filtering optics, and directed to a photodiode. The voltage generated from the photodiode contains both a DC signal and a small AC component which represents the change in the probe beam reflectivity due to the pump heating event at frequency $f$. This AC component is filtered from the DC component with lock-in amplification, and the in-phase and out-of-phase components of the AC signal are monitored as a function of pump-probe delay time, which we can monitor over $\sim 7 \mathrm{~ns}$ of delay time (although typically I find that $4-5 \mathrm{~ns}$ is sufficient to exact the thermal boundary conductance of the majority of the samples of interest). A schematic of the TDTR system in my lab at the University of Virginia is shown in Figure 3.

TDTR data taken on a $117 \mathrm{~nm}$ Al film evaporated onto a single crystalline $\mathrm{Si}$ substrate are shown in Figure 4. Characteristic in the TDTR data is a rapid rise in signal which is related to the increase in electronic temperature due to the pulse absorption followed by an exponential decay with different time constants related to the various cooling processes in the $\mathrm{Al}$ film. In the first $\sim 10 \mathrm{ps}$, the signal decay is related to the hot electron equilibration with the lattice, indicative of short pulsed laser heating $[49,61$, 70-79]. Once the electrons and phonon have equilibrated, 


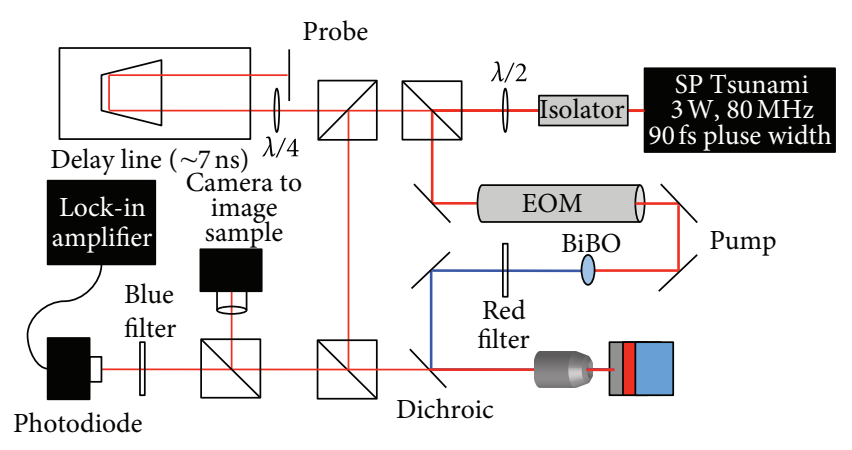

FIGURE 3: Schematic of TDTR system at the University of Virginia.

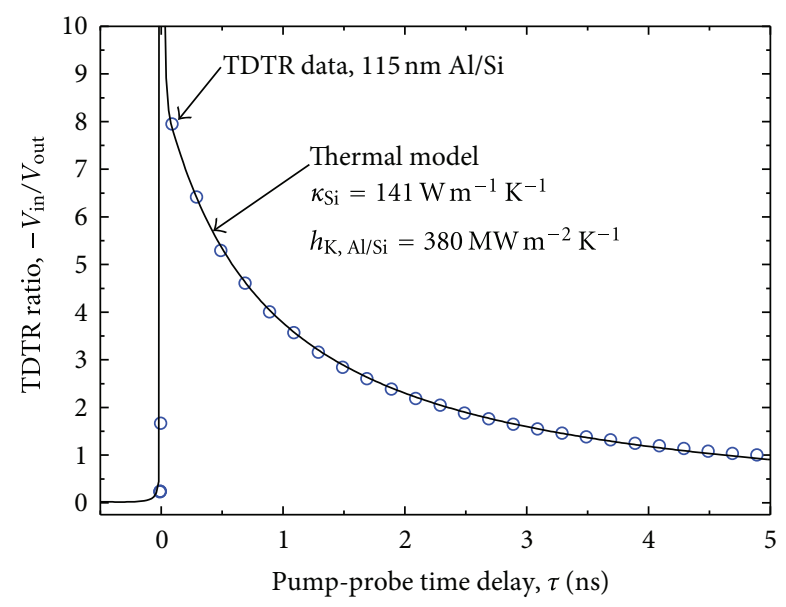

FIgURE 4: TDTR data on a $115 \mathrm{~nm}$ Al film evaporated on a $\mathrm{Si}$ substrate along with the best fit thermal model. Prior to Al evaporation, the Si was processed with an acid etch to remove the majority of the silicon native oxide layer. The thermophysical properties determined from the model best fit are $h_{\mathrm{K}}=380 \mathrm{MW} \mathrm{m}^{-2} \mathrm{~K}^{-1}$ for the thermal boundary conductance across the $\mathrm{Al} / \mathrm{Si}$ interface and $\kappa=141 \mathrm{~W} \mathrm{~m}^{-2} \mathrm{~K}^{-1}$ for the thermal conductivity of the Si substrate.

thermal diffusion through the Al film occurs, which typically occurs on a time scale of $10-100 \mathrm{ps}[48,80]$. From $100 \mathrm{ps}$ to several nanoseconds, the TDTR signal is related to the thermal boundary conductance across the $\mathrm{Al} / \mathrm{Si}$ interface and the thermal effusivity or thermal diffusivity into the Si, depending on the modulation frequency of the pump [81]. For purposes of measuring the thermal boundary conductance between two solids, the TDTR data between $\sim 100 \mathrm{ps}$ to a few nanoseconds contains the pertinent information.

To quantify the thermal boundary conductance from the TDTR data, an accurate thermal model that relates the measured lock-in output to the thermal excitation and propagation in the structures of interest must be used. For most TDTR experiments, the use of Gaussian pump and probe shapes along with a metal transducer that is thicker than the optical penetration depths facilitates the solution to the cylindrical heat equation in a multilayer structure (a metal film that is $\sim 80-120 \mathrm{~nm}$ should suffice) $[69,82,83]$. The specifics of the thermal model to predict the temperature change on the surface of the metal transducer have been outlined in several works previously $[65-67,69,84]$. It is important to note that due to the laser pulse accumulation in the sample from a Ti:Sapphire oscillator, the temperature rise on the sample surface consists of two parts: (1) a real component that represents the temperature rise due to the excitation of a single pulse and subsequent thermal diffusion from the surface of the metal into the underlying material and across the material interfaces; this real response is directly related to the time domain response of the surface temperature following the optical pump pulse; and (2) an imaginary component that represents the temperature rise from the pulse accumulation; this imaginary response is predominantly controlled by the change in surface temperature subjected to the periodic heat source at frequency $f$. Both the real and imaginary components of the heating event at the sample surface contain information about the thermal properties of the sample and can be used to analyze TDTR data for robust data analysis. The temperature rise predicted from the thermal model is related to the measured output of the lock-in amplifier during TDTR experiments via

$$
\begin{aligned}
V_{\text {in }} & =\operatorname{Re}[Z(f, \theta)], \\
V_{\text {out }} & =\operatorname{Im}[Z(f, \theta)],
\end{aligned}
$$

where $V_{\text {in }}$ is the in-phase component of the lock-in transfer function and related to the real component of the temperature rise and $V_{\text {out }}$ is the out-of-phase component of the lockin transfer function and related to the imaginary component of the temperature rise. $Z(f, \theta)$, which is the lock-in transfer function, is a function of pump modulation frequency, $f$, and surface temperature rise, $\theta$, and is given by

$$
Z(f, T)=\xi \sum_{M=-\infty}^{\infty} \theta\left(f+f_{\text {laser }}\right) \exp \left[\imath M f_{\text {laser }} \tau\right]
$$

where $\xi$ is a constant related to the electronics, thermoreflectance coefficient, laser powers, and frequency of the laser source, $f_{\text {laser }}$, and $\tau$ is the pump-probe delay time. By nature of (4), pulse accumulation is taken into account. Solving (3) for various pump-probe delay times, $\tau$, yields the output of the lock-in amplifier that is related to the measured data through the various thermal properties of the system. To analyze the data, the negative ratio of $V_{\text {in }}$ to $V_{\text {out }}\left(-V_{\text {in }} / V_{\text {out }}\right)$ yields a quantity that is directly related to the real temperature rise on the surface of the sample and inversely related to the imaginary temperature rise on the surface of the sample. Using this ratio is much more robust than using either $V_{\text {in }}$ and $V_{\text {out }}$ alone since it minimizes error due to probe beam drift and dephasing, decreases the noise due to the electronics, and eliminates the need to scale the model to the experimental data or need to know the thermoreflectance coefficient of the metal transducer $[29,85]$. In Figure 4, I show the best fit of the model to the TDTR data along with the values of $\mathrm{Al} / \mathrm{Si} h_{\mathrm{K}}$ and the Si $\kappa$ that achieve this best fit. I often use an $\mathrm{Si}$ substrate as a calibration of TDTR to ensure our experimental measurement can accurately reproduce the thermal conductivity of single crystalline silicon at room temperature $[86,87]$ (note, substantially below room temperature, 
the thermal conductivity of single crystalline silicon cannot be accurately measured due to ballistic phonon effects, which will not be discussed in this paper; however, the reader is referred to $[88,89]$ that discussed this mean free path effect in more detail).

\section{Semiclassical Formalism for Predicting Thermal Boundary Conductance}

Semiclassical modeling based on analytical expressions of the electron or phonon flux from a particle basis serves as a robust, relatively simple approach to gain fundamental insight into the underlying process driving thermal boundary conductance across solid interfaces. Although there are more rigorous approaches to model the thermal boundary conductance between two solids, such as molecular dynamics simulations [21, 90-95] or nonequilibrium Green's Function methods [96-100], due to their simplicity, semiclassical approaches can be very powerful for quick predictions that require very minimal computational expenses. Therefore, I will restrict the discussion of thermal boundary conductance modeling in this paper to semiclassical approaches and draw on specific variations of these approaches for modeling imperfect interfaces in the sections that follow.

The two most common approaches to modeling thermal boundary conductance are rooted in the assumptions of either a perfectly specular interface or a perfectly diffusive interface, resulting in specular or diffuse scattering of the electrons or phonons. Typical approaches for modeling the specular reflection of electron or phonon waves at interfaces use transmission calculations rooted in the Fresnel equations [101]. An example of this is the Acoustic Mismatch Model for predicting the thermal boundary conductance of phonons due to specular phonon wave interactions and transmission at interfaces $[102,103]$. These approaches are rooted in the assumptions that the electrons or phonons will see the interface as a perfectly specular wall. From simple analytical arguments, it is straight forward to show that this assumption breaks down at temperatures above $\sim 30 \mathrm{~K}$ for most materials [104], even in the event of perfect interfaces as was demonstrated experimentally [29]. However, in the event that imperfections or disorder are present at the interface, this specular assumption breaks down, even at temperatures as low as $1 \mathrm{~K}$ for certain interfaces [105]. In this case, the assumption of diffusive particle scattering must be employed to model the thermal transport across interfaces.

The most common and straight forward approach for modeling diffusive phonon scattering across solid interfaces and the resulting thermal boundary conductance is implementation of the Diffuse Mismatch Model (DMM) [17, 105]. The DMM is rooted in the fact that electrons or phonons scatter diffusively at the interface between two materials, and therefore the probability of the energy of these carriers to transmit energy from material 1 to material 2 is the probability of energy reflection from material 2 to material 1. Mathematically, this is expressed as

$$
\zeta_{1,2}=r_{2,1}=1-\zeta_{2,1}
$$

where $\zeta$ and $r$ are the probability of energy transmission and reflection, respectively. This diffusive transmission is then multiplied by the phonon flux in side 2 incident on the interface between material 1 and material 2 to give the transmitted phonon flux from side 1 to side 2, which, in the semiclassical formalism, is approximated by [16]

$$
q_{1}=\frac{1}{2} \sum_{j} \int_{\epsilon_{\min , j}}^{\epsilon_{\max , j}} \epsilon_{j} D_{1, j} f v_{1, j} \zeta_{1,2} d \epsilon
$$

where $j$ is an index corresponding to the energy level (or phonon mode/branch), $\epsilon$ is the energy of the carriers, $f$ is the statistical distribution function of the carriers, $v$ is the velocity of the carriers, and $\epsilon_{\min }$ and $\epsilon_{\max }$ are the minimum and maximum energy of the carrier in level $j$. From (1), in the limit of an infinitesimally small temperature rise, the semiclassical, "particle" formalism for the thermal boundary conductance across interfaces is given by

$$
h_{\mathrm{K}, 1,2}=\frac{1}{2} \sum_{j} \int_{\epsilon_{\min , j}}^{\epsilon_{\max , j}} \epsilon_{j} D_{1, j} \frac{\partial f}{\partial T} v_{1, j} \zeta_{1,2} d \epsilon,
$$

which is applicable for both electrons and phonons (and photons, for that matter). More rigorous derivations of $h_{\mathrm{K}, 1,2}$ are discussed elsewhere [95]. In addition, several works have examined the assumptions going into calculations of (7), including the use of phonon dispersion [106], realistic density of states [107], and isotropy [108-110]. The calculation of $\zeta_{1,2}$ relies on the definition of diffusive scattering (5) and the fact that a diffusive scattering event at an interface will lead to a black, thermalizing boundary. In this case, the principle of detailed balance can be evoked [111] and the DMM transmission is given by

$$
\zeta_{1,2}=\frac{q_{2,1}}{q_{1,2}+q_{2,1}}
$$

Note that this transmission expression is valid for both electrons and phonons (and, again, photons) scattering diffusively at interfaces. I will not discuss the specifics of the transmission calculations in this paper, since the goal of this paper is to review recent advances in experimental observations of thermal boundary conductance at rough interfaces. However, several additional works have described the derivation of the DMM transmission coefficient in great detail, and the reader is directed to the following references to understand the fundamental assumptions of detailed balance and how it is applied to the DMM transmission $[17,104]$, the role of inelastic scattering on this energy transmission [112-114], and the role of fluxes and transport around these interfaces $[95,115,116]$. I will discuss the modifications to (8) that have been made to describe the additional processes affecting transmission across imperfect interfaces in the sections that follow. 


\section{Thermal Transport between Two Metals: The Electron-Dominated Thermal Boundary Conductance and the Effects of Compositional Mixing}

Typically, the thermal boundary conductance in a system is only a major concern when the interfacial transport is low compared to the transport in either of the materials comprising the interface. However, at metal/metal interfaces, due to the relatively high thermal conductivity of pure metals, the transport across these interfaces can be a limiting resistance, even though the thermal boundary conductance can reach upwards of $10,000 \mathrm{MW} \mathrm{m}^{-2} \mathrm{~K}^{-1}$ at room temperature.

There have only been limited experimental measurements of the thermal boundary conductance across metal/ metal interfaces compared to those at interfaces comprised of at least one nonmetal. One of the first studies by Clemens et al. [117] reported on the thermal boundary conductance across a series of metal/metal bilayer and multilayer films. In the bilayer films, they were able to directly measure the thermal boundary conductance across $\mathrm{Ni} / \mathrm{Ti}$ and $\mathrm{Ni} / \mathrm{Zr}$ interfaces. These values, shown in Figure 5, are similar to the room temperature values of the TiN/MgO interface [29], which as discussed previously, is the highest phonon-dominated thermal boundary conductance ever measured. Gundrum et al. [28] measured the thermal boundary conductance across an interface between two free electron metals, $\mathrm{Al}$ and $\mathrm{Cu}$. The thermal boundary conductance was $\sim 4,000 \mathrm{MW} \mathrm{m}^{-2} \mathrm{~K}^{-1}$ at room temperature, half-an-order of magnitude larger than the highest phonon-dominated thermal boundary conductance ever measured. This was theorized to be due to purely free-electron dominated transport across the $\mathrm{Al} / \mathrm{Cu}$ interfaces. They derived a variation of the DMM for free electrons, given by

$$
h_{\mathrm{K}}=\frac{\gamma_{1} v_{\mathrm{F}, 1} \gamma_{2} v_{\mathrm{F}, 2}}{4\left(\gamma_{1} v_{\mathrm{F}, 1}+\gamma_{2} v_{\mathrm{F}, 2}\right)} T \text {, }
$$

where $\gamma$ is the linear coefficient to the electron heat capacity (often referred to as the Sommerfeld coefficient) [118] and $v_{\mathrm{F}}$ is the Fermi velocity (recently, we have derived a more general form of this electron DMM for nonfree electron metals [119]). Calculations of (9) for an $\mathrm{Al} / \mathrm{Cu}$ interface are shown as the line in Figure 5, which show acceptable agreement with the experimental data, alluding to the fact that this interface is dominated by free electron transport.

Recently, Wilson and Cahill [27] followed up to this $\mathrm{Al} / \mathrm{Cu}$ measurement by measuring the thermal boundary conductance deduced from thermal conductivity measurements of $\mathrm{Pd} / \mathrm{Ir}$ multilayers. These multilayers had very low electrical resistivity, and the deduced thermal boundary conductance is, to date, the highest thermal boundary conductance ever measured. Their work also demonstrated that the Wiedemann-Franz Law [120] is valid at solid interfaces [121], further proving that the transport at metal/metal interfaces is an electron-dominated phenomenon. The origin of the low thermal boundary conductance of the $\mathrm{Ni} / \mathrm{Ti}$ and $\mathrm{Ni} / \mathrm{Zr}$ interfaces compared to the $\mathrm{Pd} / \mathrm{Ir}$ and $\mathrm{Al} / \mathrm{Cu}$ interfaces is currently unknown; however, strong electron-phonon

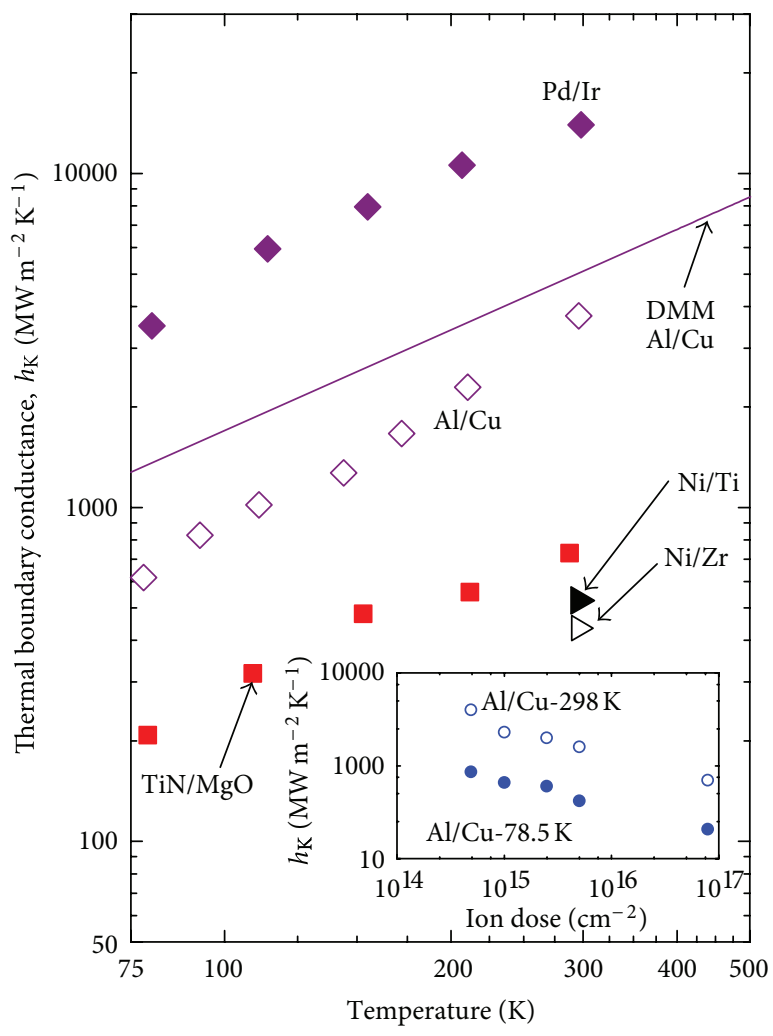

FIGURE 5: Thermal boundary conductance at metal-metal interfaces (Pd/Ir [27], Al/Cu [28], Ni/Ti [117], and Ni/Zr [117]) compared to the highest phonon-dominated thermal boundary conductance ever measured ( $\mathrm{TiN} / \mathrm{MgO}$ [29]). Inset: thermal boundary conductance at the $\mathrm{Al} / \mathrm{Cu}$ interfaces as a function of ion dose.

coupling effects in the $\mathrm{Ni}$, $\mathrm{Ti}$, and $\mathrm{Zr}$ transition metals, which would lead to high electrical resistivities compared to $\mathrm{Al}, \mathrm{Cu}$, or the $\mathrm{Pd} / \mathrm{Ir}$ multilayers, could be playing a role. However, with the recent result that the Wiedemann-Franz Law is valid at metal/metal interfaces [27], these underlying mechanisms could be deduced from electrical resistivity data.

Due to this validity of the Wiedemann-Franz law at metalmetal interfaces, disorder, which would lead to an enhancement in electron-phonon and electron-defect scattering and thereby decrease the electrical conductivity (increase the electrical resistivity), should also lead to a decrease in electron thermal boundary conductance. This was verified by Gundrum et al. [28] by ion irradiating the $\mathrm{Al} / \mathrm{Cu}$ interfaces with $1 \mathrm{MeV} \mathrm{Kr}$ ions to induce an $\mathrm{AlCu}$ intermixed layer at the interface, where the thickness of this layer would increase with increasing ion dose. As seen in the inset of Figure 5, the thermal boundary conductance across the $\mathrm{Al} / \mathrm{Cu}$ interface systematically decreases with an increase in ion dose. This is consistent with the increase in thickness of the $\mathrm{AlCu}$ intermixed layer, which leads to increased electron scattering around the interface and therefore a decrease in the thermal boundary conductance from the $\mathrm{Al}$ to the $\mathrm{Cu}$.

In this section, I reviewed the limited experimental data on thermal boundary conductance at metal/metal interfaces and the effects of $\mathrm{AlCu}$ mixing at the interface leading to 
a decrease in thermal boundary conductance. In general, metal/metal interfaces do not pose a limiting thermal resistance in most technology due to their relatively high thermal boundary conductances compared to metal/nonmetal or nonmetal/nonmetal interfaces where phonon scattering dominates transport. Therefore, for the remainder of this paper, I will focus on the effects of interfacial imperfections on phonon transport by considering different types of imperfections.

\section{Effects of Compositional Intermixing on Phonon Thermal Boundary Conductance}

In the previous section, I reviewed the effects of intermixing of $\mathrm{Al}$ and $\mathrm{Cu}$ on electron scattering and resulting thermal boundary conductance. At the $\mathrm{Al} / \mathrm{Cu}$ interface, the thermal boundary conductance is dominated by electron transport. However, at interfaces in which one material is a nonmetal, the thermal boundary conductance is dominated by phonon transport [30].

We have studied the problem of compositional intermixing on phonon thermal boundary conductance by measuring $h_{\mathrm{K}}$ across $\mathrm{Cr} / \mathrm{Si}$ interfaces with varying degrees of $\mathrm{CrSi}$ intermixed regions [22]. We accomplished this via in situ processing of the Si surface prior to $\mathrm{Cr}$ film sputtering followed by in situ heat treatments. This led to compositionally mixed regions of $\mathrm{CrSi}$ around the $\mathrm{Cr} / \mathrm{Si}$ interface that were measured with Auger Electron Spectroscopy and Transmission Electron Microscopy. We measured the thermal boundary conductance from the $\mathrm{Cr}$ to $\mathrm{Si}$ with a variation of TDTR with a regeneratively amplified laser system (repetition rate $250 \mathrm{kHz})[22,122]$. The measured thermal boundary conductance across these $\mathrm{Cr} / \mathrm{Si}$ interfaces is shown in Figure 6. The measured thermal boundary conductance decreases as the compositionally mixed layer increases. For comparison, I show the thermal boundary conductance from the $\mathrm{Al} / \mathrm{Cu}$ interfaces reported by Gundrum et al. [28] and discussed in Section 5. The dependency of the $\mathrm{Al} / \mathrm{Cu}$ electron dominated thermal boundary conductance as a function of layer thickness is similar to that at the $\mathrm{Cr} / \mathrm{Si}$ phonon dominated interface-that is, as the thickness of the compositionally mixed interfacial layer is increased, the thermal boundary conductance decreases.

To understand the underlying phonon mechanisms driving thermal boundary conductance at compositionally mixed interfacial regions, we extended the DMM for phonon transport outlined in Section 4 to a three-layer system. We have discussed this approach in detail in our previous works [123, 124]. In short, in this approach, we described the interfacial, compositionally mixed region as a thin layer of thickness $d$ with averaged properties of material 1 and material 2 comprising the interface. Therefore, for the $\mathrm{Cr} / \mathrm{Si}$ interface, the interfacial layer had averaged properties of $\mathrm{Cr}$ and $\mathrm{Si}$. The properties of this compositionally mixed interfacial layer were calculated via the virtual crystal approximation [125]. The thermal boundary conductance was then calculated by

$$
h_{\mathrm{K}, 1,2}=\left(h_{\mathrm{K}, 1, \text { int }}^{-1}+h_{\mathrm{K}, \text { int }, 2}^{-1}\right)^{-1} \text {, }
$$

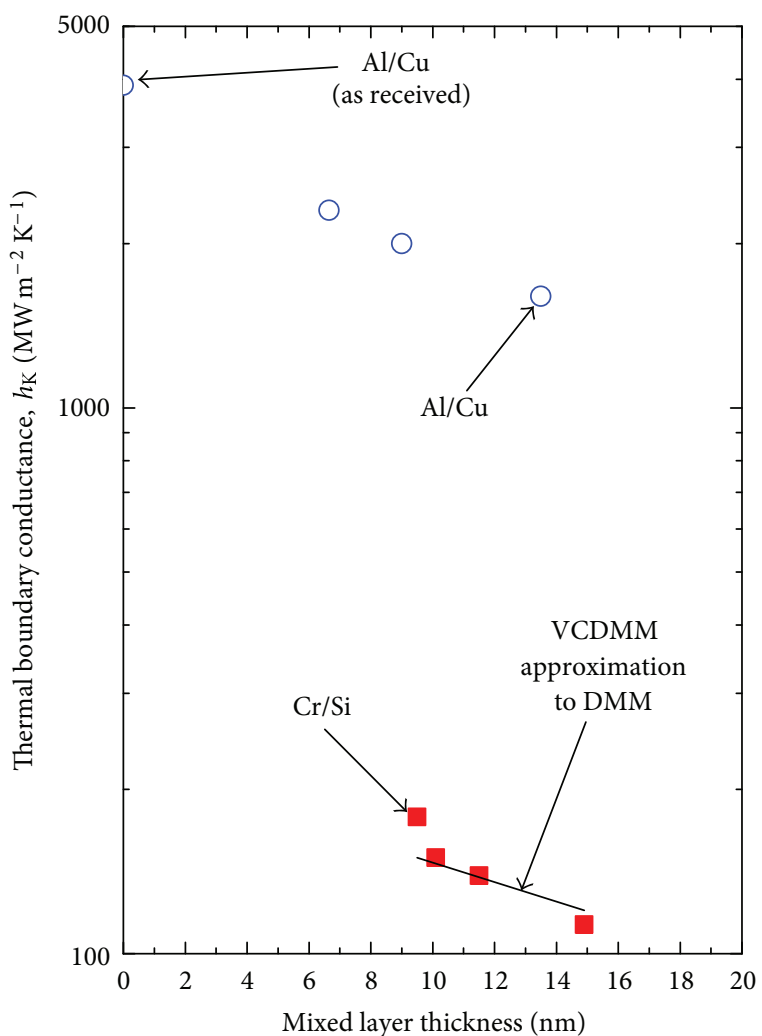

FIgURE 6: Thermal boundary conductance of a series of $\mathrm{Cr} / \mathrm{Si}$ interfaces as a function of $\mathrm{CrSi}$ mixed layer thickness [22]. For comparison, I also show the thermal boundary conductance from the $\mathrm{Al} / \mathrm{Cu}$ interfaces reported by Gundrum et al. [28] and discussed in Section 5. Compositional mixing leads to a decrease in both electron- and phonon-dominated thermal boundary conductance.

where "int" refers to the interfacial mixture, the properties which are described by a virtual crystal averaged property of material 1 and material 2 , and $h_{\mathrm{K}}$ is calculated via (7). The transmission coefficient was then calculated via (8), where the phonon fluxes in each of the materials and the interfacial layer are calculated via (6). As this model is based on the phonon DMM, we referred to this model as the Virtual Crystal DMM (VCDMM). Calculations of our VCDMM as a function of of mixed layer thickness, $d$, for a $\mathrm{Cr} / \mathrm{Si}$ interface including a varying width of a $\mathrm{CrSi}$ compositionally mixed region are shown in Figure 6. Details of these particular calculations are discussed in our previous work [22]. The model shows very good agreement with our experimentally measured data.

The calculations discussed above for the VCDMM for the $\mathrm{Cr} / \mathrm{Si}$ interface were specific for phonon interactions and scattering in a compositionally disordered CrSi layer of varying thickness $d$. Inherent to these calculations were the virtual crystal assumption of averaged layer properties based on a model derived for crystalline alloys [125]. The properties of an interfacial mixed layer can take on a range of structures and morphologies, and depending on the materials comprising the interface, the region may or may not form an alloy compound. We developed an extension to the VCDMM to account for complete disorder in an intermixed region 
(i.e., amorphous) [124]; however, in general, these approaches must be further validated. With the limited experimental data on the effects of compositional mixing on thermal boundary conductance, it is difficult to draw any concrete conclusions on either the fundamental electron or phonon mechanisms driving transport in an interfacial mixed region. However, it is apparent that the presence of this compositionally mixed interfacial region will decrease the interfacial thermal transport from one material to another.

\section{Effects of Roughness on Phonon Thermal Boundary Conductance}

The previous section discussed the effects of compositional mixing on phonon-mediated thermal boundary conductance. In this case, the interface is not compositionally abrupt but consists of some region of graded compositional properties that was referred to as a mixed layer. This begs the question, what type of thermal perturbations and phonon scattering mechanisms can exist at compositional abrupt, yet nonperfect interfaces? We studied this problem by considering thermal transport across interfaces between thin $\mathrm{Al}$ films and geometrically roughened $\mathrm{Si}$ substrates. With pre-Al-deposition Si processing, the silicon surface created a geometrically rough boundary between the $\mathrm{Al}$ and $\mathrm{Si}$. However, by ensuring that the native oxide layer reforms on the $\mathrm{Si}$ after processing, the Al will not migrate or spike into the $\mathrm{Si}$ as the native oxide layer in the $\mathrm{Si}$ acts as a diffusion barrier. This creates a compositionally abrupt, yet geometrically rough $\mathrm{Al} / \mathrm{Si}$ interface.

In a recent series of papers [19-21], we explored the extent of manipulation of phonon thermal boundary conductance through either chemical roughening of the Si $[20,21]$ or creating roughness by synthesizing $\mathrm{Si}_{1-x} \mathrm{Ge}_{x}$ quantum dots on an Si surface. In both situations, the samples were exposed to ambient atmosphere to allow the formation of a silica or germania native oxide layer after surface modification to serve as a diffusion barrier to the Al transducer, as mentioned above; this was confirmed with cross-sectional transmission electron microscopy [21]. We quantified the roughness of the various surfaces with atomic force microscopy and used the root mean square (RMS) roughness of the silicon surface $(\delta)$ as the metric for comparison. We measured the thermal boundary conductance across the $\mathrm{Al} / \mathrm{Si}$ interfaces from 100 to $300 \mathrm{~K}$ with TDTR.

The thermal boundary conductance as a function of temperature for the $\mathrm{Al} /$ chemically roughened Si interfaces [21] is shown in Figure 7 (filled symbols). In addition, I have included the Kapitza conductance at a nominally flat and oxide-free $\mathrm{Al} / \mathrm{Si}$ interface as reported in [89] (open squares). As the data indicate, even a thin native oxide layer at the interface substantially reduces the effective Kapitza conductance ( $>50 \%$ reduction at room temperature). In addition, these two datasets demonstrate significantly different temperature dependencies, suggesting that the oxide layer inhibits multiple-phonon scattering events which would otherwise contribute to Kapitza conductance [112, 113]. Similarly, comparing the four datasets of the present study,

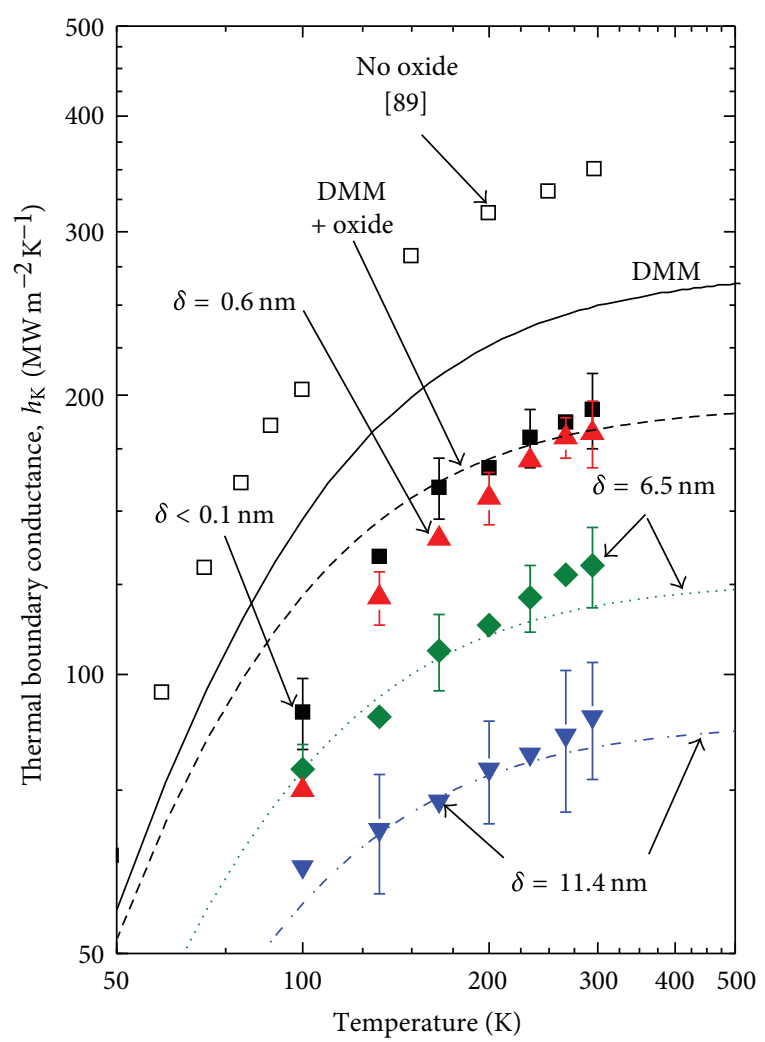

Figure 7: Predicted and measured values of Kapitza conductance at $\mathrm{Al} / \mathrm{Si}$ interfaces plotted as a function of temperature. The open circles are the measured values at oxide-free $\mathrm{Al} / \mathrm{Si}$ interfaces from [89], and the filled symbols are the data measured in our previous work [21]. It is evident that both the presence of a native oxide layer and interface roughness can have a significant effect on Kapitza conductance. Not only does roughness decrease Kapitza conductance, but also it suppresses the temperature dependence. The agreement between the dash/dot lines and the data suggests that the DMM can be adjusted to take into account both the presence of an oxide layer and interface roughness.

increased interface roughness both reduces the magnitude of Kapitza conductance as well as suppresses its temperature dependence-that is, Kapitza conductance is less temperature dependent as interface roughness increases.

In addition to the data, I have plotted several different predictive models in Figure 7. All models are calculated assuming that elastic phonon-phonon interactions dominate Kapitza conductance-that is, phonons in silicon at frequencies higher than the maximum phonon frequency of aluminum do not participate in transport. The diffuse mismatch model [17] is calculated using assumptions that we have previously outlined in [106], where the vibrational properties of film and substrate are approximated by fitting polynomials to the phonon dispersion curves of aluminum [126] and silicon [127] along the [100] crystallographic direction; assumed spherical Brillouin zones are then constructed via an isotropic revolution of these polynomial fits in wavevector space. As seen in Figure 7, the prediction of the DMM falls between the data of [89] and that at the smoothest 
interface presently considered. This suggests that without an oxide layer, inelastic phonon-phonon scattering could play a role in thermal transport across aluminum-silicon interfaces $[112,113]$. On the other hand, we attribute the difference between the predicted and measured values at the smoothest interface considered (black squares) to the native oxide layer. The conductance of this oxide layer is described by its thermal conductivity divided by its thickness,

$$
h_{\text {oxide }}=\frac{\kappa_{\text {oxide }}}{t_{\text {oxide }}} .
$$

When evaluating (12), we use the temperature-dependent bulk thermal conductivity of $\alpha: \mathrm{SiO}_{2}$, as it has been shown that the thermal conductivity of thin film $\alpha: \mathrm{SiO}_{2}$ does not substantially differ from that of bulk [29]. A series resistor approach then yields

$$
h_{\mathrm{K}}=\left(h_{\mathrm{K}, \mathrm{DMM}}^{-1}+h_{\mathrm{oxide}}^{-1}\right)^{-1} .
$$

This prediction is represented by the solid black line in Figure 7 and agrees well with our experimental data.

In order to take interfacial roughness into account, we introduce a spectral attenuation coefficient (discussed in detail in our previous work [19]) and insert this coefficient into the integral expression of the DMM. This coefficient, $\gamma$, is unity when the phonon wavelength, $\lambda$, is greater than the RMS roughness, $\delta$. On the other hand, $\gamma=\exp [-(4 \pi \beta / \lambda) \delta]$ when $\lambda<\delta$. That is, phonons with wavelengths greater than $\delta$ are unaffected by the roughness of the interface, whereas those with wavelengths less than $\delta$ are affected in a fashion similar to that of photons in an absorptive media, for example, the Beer-Lambert law. Qualitatively speaking, this approach suggests that as the "absorptivity" of the interface increases, so too does the temperature drop across it. With the spectral attenuation coefficient implemented, the DMM is once again plotted in Figure 7 for roughnesses of $6.5 \mathrm{~nm}$ and $11.4 \mathrm{~nm}$. As is evident in the plot, this approach not only captures the reduction in Kapitza conductance due to interface roughening, but also captures the reduction in temperature dependence as well.

Finally, I plot room temperature Kapitza conductance as a function of RMS roughness in Figure 8 (squares), comparing the chemically roughened data from our paper [21], the aforementioned roughness DMM model calculated at $300 \mathrm{~K}$, and two prior sets of experimental data from our previous works in which we explored other methods of roughening the silicon surface (different chemical etchants (left pointing triangles) [20] and quantum dot roughening (circles) [19]), as mentioned at the beginning of this section. Regardless of the silicon roughening procedure, the thermal boundary conductances all follows a similar relationship with RMS roughness.

To summarize, we have extensively characterized roughness effects on thermal boundary conductance across $\mathrm{Al} / \mathrm{Si}$ interfaces. In general, we found that an increase in RMS roughness leads to a systematic decrease in thermal boundary conductance. We developed an extension to the DMM that is based around phonon wavelength considerations in



Figure 8: Room-temperature predicted (line) and measured thermal boundary conductance at $\mathrm{Al} / \mathrm{Si}$ interfaces plotted as a function of interface roughness. Regardless of the silicon roughening procedure (indicated in the plot), the thermal boundary conductances all follows a similar relationship with RMS roughness.

which only certain phonon wavelengths see the interfacial roughness. This model captures both the magnitude of the experimental data and the temperature and roughness trends well. However, this RMS roughness phenomenon, to my knowledge, has only been intricately explored with wellcharacterized interfaces in our three recent works [19-21]. Different materials with different phonon spectra and oxide reactivities (e.g., gold, which does not wet a Si native oxide layer) should be explored to generalize the phonon physics at geometrically rough interfaces.

\section{Effects of Lattice Dislocations on Phonon Thermal Boundary Conductance}

Thus far, I have discussed the effects of compositional mixing and geometric roughness on thermal transport across solid interfaces. These interfacial characteristics can commonly be produced by nanoscale processing or modifying the growth/synthesis conditions. However, combinations of materials can lead to additional interfacial imperfections based on the crystal structure and atomic properties. The most common imperfections that naturally form at the interface between two solids are dislocations produced from strain relaxation that stem from the lattice mismatch between two materials. Dislocations, which can readily scatter phonons and add to thermal resistance in solids [128], have been shown to cause drastic reductions in the thermal conductivity of thin films and nanostructures. For example, at large periodic thicknesses $(\sim 10 \mathrm{~nm})$, the thermal conductivity of $\mathrm{Si} / \mathrm{Ge}$ superlattices has been shown to suddenly drop by a factor of two due to dislocation formation from strain relaxation of the Si or Ge layers on Ge or Si, respectively, leading to poor crystalline quality [129]. Similarly, threading dislocation densities of $4 \times 10^{10} \mathrm{~cm}^{-1}$ have led to AlN films on $\mathrm{SiC}$ substrates to exhibit thermal conductivities 
less than the theoretical minimum limit [130, 131]. As these dislocations stem from interfacial growth and lattice relaxation, understanding how dislocations affect thermal boundary conductance adds an important piece to the study of the phonon mechanisms affecting thermal transport across imperfect interfaces.

This issue was addressed in part by Costescu et al. [29], who measured the thermal conductivity across $\mathrm{TiN} / \mathrm{Al}_{2} \mathrm{O}_{3}$ interfaces in addition to TiN/MgO interfaces (one of the $\mathrm{TiN} / \mathrm{MgO}$ samples that was measured is shown in Figure 2). The measured $\mathrm{TiN} / \mathrm{MgO}$ (001), which is essentially free of misfit dislocations, TiN/MgO (111), which contains a high density of stacking faults but few misfit dislocations, and $\mathrm{TiN} / \mathrm{Al}_{2} \mathrm{O}_{3}$, which should contain a large density of both stacking faults and misfit dislocations, show no appreciable difference in the thermal boundary conductance. This indicates that in these well acoustically matched materials, the dislocation densities did not affect the phonon transport. However, without quantifying the dislocation density, it is difficult to understand the effects of these dislocations on thermal boundary conductance. In addition, without quantification, this does not add any direct insight into previous studies observing drastically reduced thermal conductivities of thin films due to dislocation formation (such as the AlN thin film example above [130]).

We quantitatively studied the effects of dislocation density on thermal boundary conductance by measuring the thermal boundary conductance across well-characterized $\mathrm{GaSb} / \mathrm{GaAs}$ interfaces, as is outlined in detail in our previous work [23]. The lattice mismatch between GaSb and GaAs is $7.78 \%$, and the critical thickness for strain relaxation is formed within the first monolayer. Different epitaxial growth modes were utilized to change the dislocation densities at the $\mathrm{GaSb} / \mathrm{GaAs}$ interfaces, as characterized with plan view and cross-sectional transmission electron microscopy [132, 133]. These growth techniques were able to produce $\mathrm{GaSb} / \mathrm{GaAs}$ interfaces with two ranges of dislocations densities: $5 \times 10^{6}-$ $5 \times 10^{8}$ dislocations $\mathrm{cm}^{-2}$ and $10^{9}-10^{11}$ dislocations $\mathrm{cm}^{-2}$. We measured the thermal boundary conductances across the GaSb/GaAs interfaces from 100-500 K with TDTR.

The thermal boundary conductances as a function of temperature for the two GaSb/GaAs interfaces with different dislocation densities are shown in Figure 9 (filled and open triangles for the relatively low and high dislocation dense samples, resp.). It is interesting to note that roughly a two order of magnitude change in dislocation density only leads to a factor of two change in thermal boundary conductance. To put this into perspective, at similar dislocation levels, for two orders of magnitude change in dislocation density, the thermal conductivity of GaN drops by an order of magnitude [130]. This indicates the seemingly small effect that changes in dislocation densities have on thermal boundary conductance across heavily dislocated interfaces. For comparison, I also plot the thermal boundary conductance of $\mathrm{TiN} / \mathrm{MgO}$ [29] and $\mathrm{Bi} /$ diamond [30] interfaces (as discussed in Section 2, TiN/MgO and $\mathrm{Bi} /$ diamond represent the highest and lowest phonon-mediated thermal boundary conductances ever measured). The GaSb/GaAs dislocation

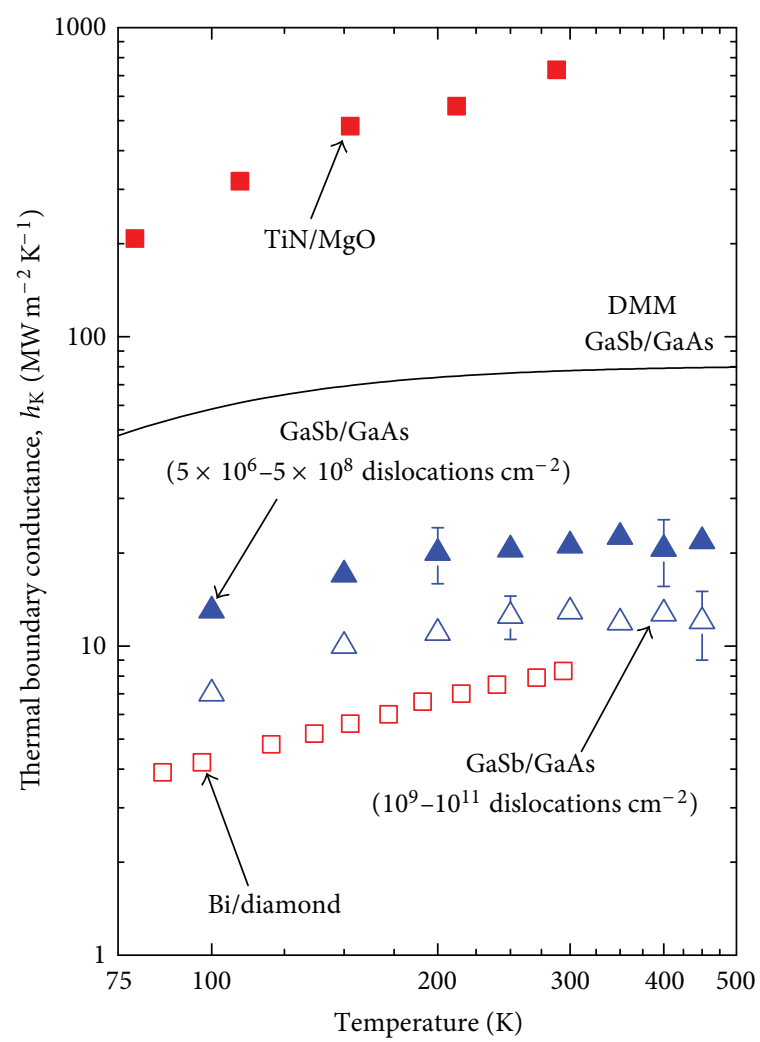

Figure 9: Thermal boundary conductances at the two different dislocation dense $\mathrm{GaSb} / \mathrm{GaAs}$ interfaces from our previous work [23]. These interfaces have dislocation densities ranging from $5 \times$ $10^{6}-5 \times 10^{8}$ dislocations $\mathrm{cm}^{-2}$ and $10^{9}-10^{11}$ dislocations $\mathrm{cm}^{-2}$. For comparison, the plot includes previously measured data at TiN/MgO [29] and Bi/diamond [30] interfaces. DMM calculations are shown for the $\mathrm{GaSb} / \mathrm{GaAs}$ interfaces, which drastically overpredict the measured data since the traditional formulation of the DMM does not account for dislocations interfaces. It is also interesting to note that roughly a two order of magnitude change in dislocation density only leads to a factor of two change in thermal boundary conductance.

dense interfaces are approaching the measured values of $\mathrm{Bi}$ /diamond. However, $\mathrm{Bi}$ and diamond have drastically different phonon spectra and are extremely acoustically mismatched (over an order of magnitude different acoustic velocities and Debye Temperatures) [134]. Based on DMM theory, acoustically mismatched interfaces will have lower phonon transmission probability than acoustically matched interfaces [17]. GaSb and GaAs have similar phonon spectra and phonon velocities and are well acoustically matched. The TiN/MgO interfaces are well acoustically matched and were epitaxially grown (like the GaSb). The differences in thermal boundary conductances between the GaSb/GaAs and $\mathrm{TiN} / \mathrm{MgO}$ well acoustically matched interfaces are over an order of magnitude, indicating the potential strong effect that dislocations can have on phonon transmission. This is further exemplified by comparing our measured thermal boundary conductances across the $\mathrm{GaSb} / \mathrm{GaAs}$ interfaces to DMM calculations for GaSb/GaAs. For these calculations, 
we used the approach that we outlined previously in which we fit a polynomial to a measured or simulated phonon dispersion in one crystallographic direction and assume an isotropic medium to calculate the DMM [106]. For DMM calculations, we used the dispersion in the $\Gamma \rightarrow \mathrm{X}$ direction of the Brillouin zone from [135] for GaSb and [136] for GaAs (we include the optical branches of GaSb and GaAs in our calculations) [137]. I plot these calculations of the $\mathrm{DMM}$ for $\mathrm{GaSb} / \mathrm{GaAs}$ as the solid line in Figure 9. Clearly, the DMM greatly overpredicts the measured values. As the DMM assumes a perfect interface and a single-phonon scattering event, this approach is not valid for the dislocation dense $\mathrm{GaSb} / \mathrm{GaAs}$ interfaces.

The discussion in this section demonstrates the detrimental effects that dislocations can have on thermal boundary conductance. However, due to the limited data, the fundamental mechanisms driving phonon dislocation interactions around interfaces are unknown. One outstanding question is the functional form of the dependency of thermal boundary conductance on dislocation density. In the thermal conductivity of a homogeneous material, the thermal conductivity is inversely proportional to the square root of the dislocation density [130]. This implies that changes in dislocation densities at a dislocation light interface will be greater than at a dislocation dense interface. Assuming the $\mathrm{GaSb} / \mathrm{GaAs}$ interface is in the dislocation dense regime, this could explain the relatively small change in thermal boundary conductance (factor of two) with a two order of magnitude change in dislocation density. However, as discussed by Costescu et al. [29], there is essentially no difference in thermal boundary conductance between TiN/MgO (001), $\mathrm{TiN} / \mathrm{MgO}$ (111), and $\mathrm{TiN} / \mathrm{Al}_{2} \mathrm{O}_{3}$, which has varying levels of dislocation densities. This implies that either the dislocation densities, although varying, were extremely low at these TiNbased interfaces; however, without exact characterization of the defect densities at these interfaces, quantitative analyses are not possible. Therefore, to elucidate the underlying role of dislocations on phonon thermal boundary conductance, controlled experiments of thin films on substrates with varying degrees of dislocation formation at the film substrate boundaries need to be pursued.

\section{Effect of Bonding on Phonon Thermal Boundary Conductance}

Thus far, I have discussed the roles that imperfections in the lattice structure or order of the masses around solid interfaces have on thermal boundary conductance. However, phonon transport is also affected by the strength of the interatomic bonding. The question then arises: what effect does interfacial bond strength have on phonon thermal boundary conductance? Very recently, this has been an active area of research in nanoscale heat transport. Several works have investigated this problem computationally or theoretically $[92,93,138-$ 142]. Experimentally, the effects of bond strength on thermal boundary conductance have been pin-pointed in a few specific systems using self-assembled monolayers, diamond anvil technologies, or surface termination to tune the interfacial interactions. Hsieh et al. [143] used a diamond anvil cell to vary the pressure at $\mathrm{Al} / \mathrm{SiC}$ interfaces and demonstrated an increase in thermal boundary conductance with pressure when an acoustically soft layer is placed between the $\mathrm{Al}$ and $\mathrm{SiC}$ (such as graphene, which interacts with the $\mathrm{Al}$ via van der Waal interactions, or $\mathrm{SiO}_{x}$, which has as low acoustic impedance). The stiffness of these "weak" interfaces increased with pressure, and a nearly order of magnitude increase in thermal boundary conductance between the $\mathrm{Al}$ and $\mathrm{SiC}$ was demonstrated at pressures of $\sim 10 \mathrm{GPa}$ compared to no applied pressure. Losego et al. [144] tuned the bonding between $\mathrm{Au}$ and quartz by using self-assembled monolayers (SAMs) sandwiched at the interface. The end group chemistries of the SAMs covalently bonded to the quartz gave a method of tuning the SAM/Au interaction strength. This led to tunability in the thermal transport from the $\mathrm{Au}$ to the quartz by increasing the density of covalent bonds that bridge the Au to the substrate. Collins and Chen [145] and Monachon and Weber [146] demonstrated the use of surface termination of diamond as a means to increasing the thermal boundary conductance between various metals and diamond substrates. Most notably, they found that oxygen termination can increase the thermal boundary conductance between $\mathrm{Al}$ and diamond by a factor of two (Collins and Chen [145]) and $\mathrm{Cr}$ and diamond by a factor of 11 (Monachon and Weber [146]) at room temperature as compared to untreated diamond.

We investigated this problem by utilizing adsorbed elemental functional groups at graphene contacts [24]. As previously mentioned, graphene interfaces are typically described as weakly interacting due to the van der Waal forces that are characteristic of cross plane graphene/graphite. Using low pressure electron beam generated plasmas [147, 148], a series of graphene films on $\mathrm{SiO}_{2} / \mathrm{Si}$ substrates were functionalized with $\mathrm{O}_{2}$. After plasma functionalization, the graphene surfaces were coated with $\mathrm{Al}$ and we measured the thermal boundary conductance between the $\mathrm{Al}$ and graphene. I show the thermal boundary conductance between $\mathrm{Al} /$ single layer graphene (SLG) and Al/oxygen functionalized SLG (Al/OSLG) in Figure 10. We observed a factor of two increase in $h_{\mathrm{K}}$ across the Al/O-SLG interface as compared with the $\mathrm{Al} / \mathrm{SLG}$ interface. We attributed this behavior to the enhanced reactivity of the oxygenated surface, which is consistent with the increase observed across the $\mathrm{Al} / \mathrm{O}$-diamond interface as compared to the $\mathrm{Al} /$ diamond interface $[145,149]$, also shown in Figure 10. The presence of oxygen-containing functional groups increases the surface energy and thus makes graphene more reactive, allowing a lower energy bonding state with the evaporated $\mathrm{Al}$ film as compared to the untreated SLG which is chemically inert. This lower energy bonding state promotes $\mathrm{Al}-\mathrm{O}$ formation, leading to a stronger Al-O-SLG junction and thereby an increase in $h_{\mathrm{K}}$.

To quantify this, we turn to the DMM, discussed in Section 4. However, in this case, the transmission coefficient must be rederived to account for the two-dimensional nature of the graphene. To calculate the transmission coefficient, we used the assumption that we discussed in a previous work [110], in which we treat the graphene sheet as a twodimensional solid, thereby performing detailed balance on 


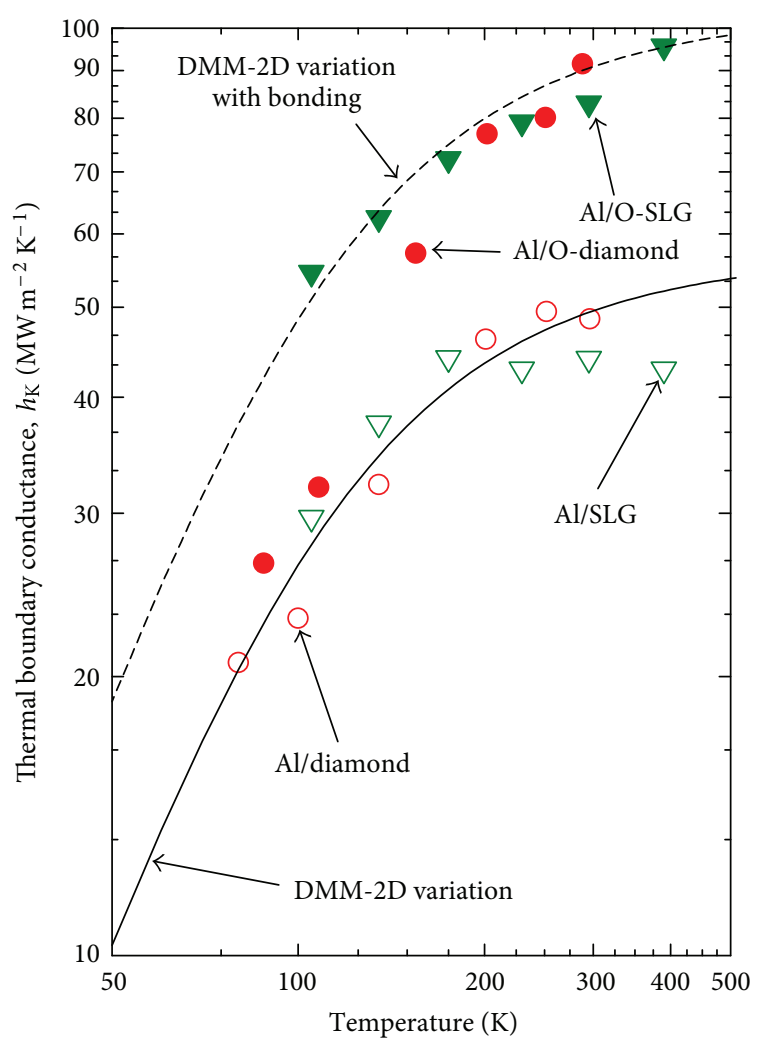

FIgURE 10: Thermal boundary conductance across our Al/SLG (filled triangles) and Al/O-SLG (unfilled triangles) interfaces [24]. The thermal transport across the Al/SLG interface increases by a factor of two with oxygen functionalization of the graphene. Our measured values are in good agreement with conductances measured across $\mathrm{Al} /$ diamond and $\mathrm{Al} / \mathrm{O}$-diamond interfaces, which exhibit an increase in thermal boundary conductance with oxygen termination $[145,149]$. We model the thermal conductance across the Al/SLG interface with the DMM, as shown by the solid line. We adjust the velocity of the SLG in our DMM calculations to model the $\mathrm{Al} / \mathrm{O}-\mathrm{SLG}$ thermal boundary conductance (dashed line), and find that the resultant velocity in the oxygenated SLG is about a factor of two higher than the non-functionalized sample. This is indicative of the increase in bond strength between the $\mathrm{Al}$ and the SLG via the oxygen adsorbates.

the fluxes in the $\mathrm{Al}$ and SLG. For the flux in the $\mathrm{Al}$, we can make the usual three-dimensional isotropic approximation of phonon flux discussed in Section 4. However, given the two-dimensional nature of the SLG, the phonon flux in this material is given by [110]

$$
q_{\mathrm{SLG}}=\frac{1}{2 a} \sum_{j} \int_{0}^{\omega_{j, \max }} \hbar \omega D_{2 \mathrm{D}, \mathrm{SLG}} f v_{\mathrm{SLG}} d \omega,
$$

where $a$ is the interlayer spacing of graphite (i.e., $=3.35 \AA$ ) [150]. For calculations of this anisotropic SLG-modified DMM (which we refer to in Figure 10 as "DMM-2D variation"), we fit polynomials to the phonon dispersions of $\mathrm{Al}$ in the $\Gamma \rightarrow \mathrm{X}$ direction [126] and of graphene in the $\Gamma \rightarrow \mathrm{K}$ direction [151] to give greater accuracy in the DMM calculations as compared to the Debye approximation [106].
We note, however, that the modes in the $\Gamma \rightarrow \mathrm{K}$ direction in graphene are extremely Debye-like (i.e., non dispersive) in the frequency regime that is elastically accessible to the $\mathrm{Al}$ (frequencies below $10 \mathrm{THz}$ ). We ignore the $\mathrm{ZA}$ mode in the graphene dispersion since the ZA mode is heavily suppressed in encased and supported SLG [43, 152]. Given that our measurements represented the cross plane conductance of $\mathrm{Al} / \mathrm{SLG}$, the cross plane velocity of SLG is meaningless. Therefore, we define $v_{\text {SLG }}$ as a fitting parameter that we assume is constant with frequency. This is a similar approach as performed in the analysis of Koh et al. [153] in which they adjust the transmission coefficient to fit the DMM to their Au/Ti/SLG data and Schmidt et al. [154] in which they adjust the phonon transmission to fit the DMM to their metal/graphite data. We note that this approach of adjusting only $v_{\text {SLG }}$ gives us more direct insight into how the bonding at the Al/SLG interface changes due to the functionalization since we are not adjusting any aspect of the graphene dispersion, only the transport velocity. The fits of the DMM are shown in Figure 10. The solid line is the DMM calculation assuming the graphene cross plane velocities are 2,455 and 1,480 $\mathrm{m} \mathrm{s}^{-1}$ ) for the longitudinal and transverse modes of the SLG, respectively (labeled "DMM$2 \mathrm{D}$ variation"). The dashed line is the DMM calculation assuming 4,687 and $2,825 \mathrm{~m} \mathrm{~s}^{-1}$ for the longitudinal and transverse modes of the O-SLG, respectively. The velocities that result in the best fit of the DMM to the SLG data are in good agreement with the cross plane velocity of bulk graphite [150], which could be indicative of similar bonding between the $\mathrm{Al}$ and SLG as the van der Waals bonds cross plane in graphite. The velocities resulting in the best fit in the $\mathrm{Al} / \mathrm{O}-$ SLG data are nearly a factor of two higher, which we attribute to the increased bond strength between the Al and SLG due to the presence of oxygen leading to covalent bonding between the $\mathrm{Al}$ and O-SLG. It is important to note that we use the velocity parameter and fitting only to lend insight into the nature of the bonding at the two different interfaces. Since the velocity is proportional to the square root of the spring constant, the increase by a factor of two in the best-fit velocity with oxygen functionalization implies that increase in thermal transport is due to an increase in interfacial bond strength by roughly $40 \%$.

In summary, in this section I have discussed the role of interfacial bonding on thermal boundary conductance at solid interfaces. This has been a topic of several recent works, as outlined in detailed in this section. The strength of the interfacial bond can be manipulated to directly tune the interfacial conductance, offering a unique "knob" for thermal transport. In our specific work outlined in [24], we showed that the conductance of a single layer graphene interface can be manipulated by elemental adsorbates from low energy plasma functionalization. It is interesting to note that the temperature dependency of the thermal boundary conductance also changes, which could be evidence of higher order phonon interactions, such as 3- or 4-phonon conversion processes at the interface [138]. However, the exact phonon conversion mechanisms occurring at variably bonded interfaces are unclear, and more research must be 
pursued to elucidate the fundamental phonon interaction problem.

\section{Summary}

The bottle neck of many modern technologies and energy solutions has boiled down to thermal engineering problem on the nanoscale. As both increases and decreases in thermal conductivity are required for different applications, the ability to control and tune the thermal properties of materials is therefore the ultimate goal to improve both device efficiency and energy consumption trends. This desired thermal control in nanosystems, however, is not a trivial task. Due to the magnitudes of the thermal mean free paths approaching or overpassing typical length scales in nanomaterials (i.e., materials with length scales less than 1 micron), the thermal transport across interfaces can dictate the overall resistance in nanosystems. However, the fundamental mechanisms driving these electron and phonon interactions at nanoscale interfaces are difficult to predict and control since the thermal boundary conductance across interfaces (often called the Kapitza conductance), $h_{\mathrm{K}}$, is intimately related to the characteristics of the interface (structure, bonding, geometry, etc.) in addition to the fundamental atomistic properties of the materials comprising the interface itself. This interplay between interfacial properties and thermal boundary conductance can lead to additional carrier scattering mechanisms and phenomena that will not only change the thermal processes but can be used to systematically manipulate and control thermal transport in nanosystems.

In this paper, I have reviewed recent experimental progress in understanding this interplay between interfacial properties on the atomic scale and thermal transport across solid interfaces. I focused this discussion specifically on the role of interfacial nanoscale "imperfections," such as surface roughness, compositional disorder, atomic dislocations, or interfacial bonding. In general, these interfacial imperfections lead to decreases in thermal boundary conductance, where each type of imperfection leads to different scattering mechanisms that can be used to control the thermal boundary conductance. This offers a unique avenue for controlling scattering and thermal transport in nanotechnology.

\section{Acknowledgments}

The author is grateful to the support from the National Science Foundation (CBET Grant no. 1134311) and Sandia National Laboratories through the LDRD program office.

\section{References}

[1] Lawrence Livermore National Laboratory, Livermore, Calif, USA 94550, LLNL Energy Flow Chart, https://flowcharts.llnl .gov/.

[2] "EPA report on clean energy and air emissions [online]," http://www.epa.gov/cleanenergy/energy-and-you/affect/airemissions.html.

[3] G. E. Moore, "Cramming more components onto integrated circuits," Electronics Magazine, vol. 38, p. 4, 1965.
[4] E. Pop, "Energy dissipation and transport in nanoscale devices," Nano Research, vol. 3, no. 3, pp. 147-169, 2010.

[5] D. G. Cahill, W. K. Ford, K. E. Goodson et al., "Nanoscale thermal transport," Journal of Applied Physics, vol. 93, no. 2, pp. 793-818, 2003.

[6] T. Hendricks and W. T. Choate, "Engineering scoping study of thermoelectric generator systems for industrial waste heat recovery," Tech. Rep., Pacific Northwest National Laboratory and BCS, 2006.

[7] K. Yazawa and A. Shakouri, "Cost-efficiency trade-off and the design of thermoelectric power generators," Environmental Science and Technology, vol. 45, pp. 7548-7553, 2011.

[8] M. S. Dresselhaus, G. Dresselhaus, X. Sun et al., "The promise of low-dimensional thermoelectric materials," Microscale Thermophysical Engineering, vol. 3, no. 2, pp. 89-100, 1999.

[9] B. K. Nayak, V. V. Iyengar, and M. C. Gupta, "Efficient light trapping in silicon solar cells by ultrafast-laser-induced selfassembled micro/nano structures," Progress in Photovoltaics, vol. 19, pp. 631-639, 2011.

[10] T. Kietzke, "Recent advances in organic solar cells," Advances in OptoElectronics, vol. 2007, Article ID 40285, 15 pages, 2007.

[11] A. N. Smith and J. P. Calame, "Impact of thin film thermophysical properties on thermal management of wide bandgap solidstate transistors," International Journal of Thermophysics, vol. 25, no. 2, pp. 409-422, 2004.

[12] G. J. Snyder and E. S. Toberer, "Complex thermoelectric materials," Nature Materials, vol. 7, no. 2, pp. 105-114, 2008.

[13] B. Poudel, Q. Hao, Y. Ma et al., "High-thermoelectric performance of nanostructured bismuth antimony telluride bulk alloys," Science, vol. 320, no. 5876, pp. 634-638, 2008.

[14] A. I. Hochbaum, R. Chen, R. D. Delgado et al., "Enhanced thermoelectric performance of rough silicon nanowires," Nature, vol. 451, no. 7175, pp. 163-167, 2008.

[15] A. I. Boukai, Y. Bunimovich, J. Tahir-Kheli, J. K. Yu, W. A. Goddard, and J. R. Heath, "Silicon nanowires as efficient thermoelectric materials," Nature, vol. 451, no. 7175, pp. 168-171, 2008.

[16] G. Chen, Nanoscale Energy Transport and Conversion: A Parallel Treatment of Electrons, Molecules, Phonons, and Photons, Oxford University Press, New York, NY, USA, 2005.

[17] E. T. Swartz and R. O. Pohl, "Thermal boundary resistance," Reviews of Modern Physics, vol. 61, no. 3, pp. 605-668, 1989.

[18] P. L. Kapitza, “The study of heat transfer in Helium II," Zhurnal Eksperimental'noi $i$ Teoreticheskoi Fiziki, p. 1, 1941.

[19] P. E. Hopkins, J. C. Duda, C. W. Petz, and J. A. Floro, "Controlling thermal conductance through quantum dot roughening at interfaces," Physical Review B, vol. 84, no. 3, Article ID 035438, 7 pages, 2011.

[20] P. E. Hopkins, L. M. Phinney, J. R. Serrano, and T. E. Beechem, "Effects of surface roughness and oxide layer on the thermal boundary conductance at aluminum/silicon interfaces," Physical Review B, vol. 82, no. 8, Article ID 085307, 5 pages, 2010.

[21] J. C. Duda and P. E. Hopkins, "Systematically controlling Kapitza conductance via chemical etching," Applied Physics Letters, vol. 100, no. 11, Article ID 111602, 4 pages, 2012.

[22] P. E. Hopkins, P. M. Norris, R. J. Stevens, T. E. Beechem, and S. Graham, "Influence of interfacial mixing on thermal boundary conductance across a chromium/silicon interface," Journal of Heat Transfer, vol. 130, no. 6, Article ID 062401, 10 pages, 2008.

[23] P. E. Hopkins, J. C. Duda, S. P. Clark et al., "Effect of dislocation density on thermal boundary conductance across $\mathrm{GaSb} / \mathrm{GaAs}$ 
interfaces," Applied Physics Letters, vol. 98, no. 16, Article ID 161913, 3 pages, 2011

[24] P. E. Hopkins, M. Baraket, E. V. Barnat et al., "Manipulating thermal conductance at metal-graphene contacts via chemical functionalization," Nano Letters, vol. 12, pp. 590-595, 2012.

[25] D. G. Cahill, K. Goodson, and A. Majumdar, "Thermometry and thermal transport in micro/nanoscale solid-state devices and structures," Journal of Heat Transfer, vol. 124, no. 2, pp. 223-241, 2002.

[26] D. G. Cahill, "Extremes of heat conduction? Pushing the boundaries of the thermal conductivity of materials," MRS Bulletin, vol. 37, pp. 855-863, 2012.

[27] R. B. Wilson and D. G. Cahill, "Experimental validation of the interfacial form of the wiedemann-franz law," Physical Review Letters, vol. 108, Article ID 255901, 5 pages, 2012.

[28] B. C. Gundrum, D. G. Cahill, and R. S. Averback, "Thermal conductance of metal-metal interfaces," Physical Review B, vol. 72, no. 24, 5 pages, 2005.

[29] R. M. Costescu, M. A. Wall, and D. G. Cahill, "Thermal conductance of epitaxial interfaces," Physical Review B, vol. 67, no. 5, Article ID 054302, 5 pages, 2003.

[30] H. K. Lyeo and D. G. Cahill, "Thermal conductance of interfaces between highly dissimilar materials," Physical Review B, vol. 73, no. 14, Article ID 144301, 6 pages, 2006.

[31] D. G. Cahill, "Thermal conductivity measurement from 30 to 750 K: the $3 \omega$ method," Review of Scientific Instruments, vol. 61, no. 2, article 802, 7 pages, 1990.

[32] Y. C. Tai, C. H. Mastrangelo, and R. S. Muller, "Thermal conductivity of heavily doped low-pressure chemical vapor deposited polycrystalline silicon films," Journal of Applied Physics, vol. 63, no. 5, article 1442, 6 pages, 1988.

[33] L. M. Phinney, E. S. Piekos, and J. D. Kuppers, "Bond pad effects on steady state thermal conductivity measurement using suspended micromachined test structures," in Proceedings of the ASME International Mechanical Engineering Congress and Exposition (IMECE '07), vol. 41349, Seattle, Wash, USA, 2007.

[34] S. Uma, A. D. McConnell, M. Asheghi, K. Kurabayashi, and K. E. Goodson, "Temperature-dependent thermal conductivity of undoped polycrystalline silicon layers," International Journal of Thermophysics, vol. 22, no. 2, pp. 605-616, 2001.

[35] A. D. McConnell, S. Uma, and K. E. Goodson, "Thermal conductivity of doped polysilicon layers," Journal of Microelectromechanical Systems, vol. 10, no. 3, pp. 360-369, 2001.

[36] D. Li, Y. Wu, P. Kim, L. Shi, P. Yang, and A. Majumdar, "Thermal conductivity of individual silicon nanowires," Applied Physics Letters, vol. 83, no. 14, article 2934, 3 pages, 2003.

[37] Z. Chen, W. Jang, W. Bao, C. N. Lau, and C. Dames, "Thermal contact resistance between graphene and silicon dioxide," Applied Physics Letters, vol. 95, no. 16, Article ID 161910, 3 pages, 2009.

[38] L. Lu, W. Yi, and D. L. Zhang, "3 $\omega$ method for specific heat and thermal conductivity measurements," Review of Scientific Instruments, vol. 72, no. 7, pp. 2996-3003, 2001.

[39] T. Tong and A. Majumdar, "Reexamining the 3-omega technique for thin film thermal characterization," Review of Scientific Instruments, vol. 77, no. 10, Article ID 104902, 9 pages, 2006.

[40] M. L. Bauer, C. M. Bauer, M. C. Fish et al., "Thin-film aerogel thermal conductivity measurements via $3 \omega$," Journal of NonCrystalline Solids, vol. 357, no. 15, pp. 2960-2965, 2011.
[41] B. W. Olson, S. Graham, and K. Chen, "A practical extension of the $3 \omega$ method to multilayer structures," Review of Scientific Instruments, vol. 76, no. 5, Article ID 053901, 7 pages, 2005.

[42] P. E. Hopkins and L. M. Phinney, "Thermal conductivity measurements on polycrystalline silicon microbridges using the $3 \omega$ technique," Journal of Heat Transfer, vol. 131, no. 4, Article ID 043201, 8 pages, 2009.

[43] J. H. Seol, I. Jo, A. L. Moore et al., "Two-dimensional phonon transport in supported graphene," Science, vol. 328, no. 5975, pp. 213-216, 2010.

[44] L. Shi, D. Li, C. Yu et al., "Measuring thermal and thermoelectric properties of one-dimensional nanostructures using a microfabricated device," Journal of Heat Transfer, vol. 125, no. 5, pp. 881-888, 2003.

[45] H. S. Carslaw and J. C. Jaeger, Conduction of Heat in Solids, Oxford University Press, New York, NY, US, 2nd edition, 1959.

[46] H. W. Deem and W. D. Wood, "Flash thermal-diffusivity measurements using a laser," Review of Scientific Instruments, vol. 33, no. 10, pp. 1107-1109, 1962.

[47] J. Guo, X. Wang, and T. Wang, "Thermal characterization of microscale conductive and nonconductive wires using transient electrothermal technique," Journal of Applied Physics, vol. 101, no. 6, Article ID 063537, 7 pages, 2007.

[48] C. A. Paddock and G. L. Eesley, "Transient thermoreflectance from thin metal films," Journal of Applied Physics, vol. 60, no. 1, pp. 285-290, 1986.

[49] G. L. Eesley, "Observation of nonequilibrium electron heating in copper," Physical Review Letters, vol. 51, no. 23, pp. 2140-2143, 1983.

[50] J. H. Weaver, D. W. Lynch, C. H. Culp, and R. Rosei, "Thermoreflectance of V, Nb, and paramagnetic Cr," Physical Review $B$, vol. 14, no. 2, pp. 459-463, 1976.

[51] E. Colavita, A. Franciosi, D. W. Lynch, G. Paolucci, and R. Rosei, "Thermoreflectance investigation of the antiferromagnetic and paramagnetic phases of Cr," Physical Review B, vol. 27, no. 3, pp. 1653-1663, 1983

[52] E. Colavita, A. Franciosi, C. Mariani, and R. Rosei, "Thermoreflectance test of W, Mo, and paramagnetic Cr band structures," Physical Review B, vol. 27, no. 8, pp. 4684-4693, 1983.

[53] D. W. Lynch, R. Rosei, and J. H. Weaver, "Infrared and visible optical properties of single crystal Ni at $4 \mathrm{~K}$," Solid State Communications, vol. 9, no. 24, pp. 2195-2199, 1971.

[54] R. Rosei and D. W. Lynch, "Thermomodulation spectra of Al, $\mathrm{Au}$, and Cu," Physical Review B, vol. 5, no. 10, pp. 3883-3894, 1972.

[55] R. Rosei, C. H. Culp, and J. H. Weaver, “Temperature modulation of the optical transitions involving the fermi surface in Ag: experimental," Physical Review B, vol. 10, no. 2, pp. 484-489, 1974.

[56] R. Rosei, "Temperature modulation of the optical transitions involving the fermi surface in Ag: theory," Physical Review B, vol. 10, no. 2, pp. 474-483, 1974.

[57] J. L. Hostetler, A. N. Smith, and P. M. Norris, “Thin-film thermal conductivity and thickness measurements using picosecond ultrasonics," Microscale Thermophysical Engineering, vol. 1, no. 3, pp. 237-244, 1997.

[58] W. P. Hsieh and D. G. Cahill, "Ta and $\mathrm{Au}(\mathrm{Pd})$ alloy metal film transducers for time-domain thermoreflectance at high pressures," Journal of Applied Physics, vol. 109, no. 11, Article ID 113520, 4 pages, 2011. 
[59] Y. Wang, J. Y. Park, Y. K. Koh, and D. G. Cahill, "Thermoreflectance of metal transducers for time-domain thermoreflectance," Journal of Applied Physics, vol. 108, no. 4, Article ID 043507, 4 pages, 2010.

[60] P. E. Hopkins, "Effects of electron-boundary scattering on changes in thermoreflectance in thin metal films undergoing intraband excitations," Journal of Applied Physics, vol. 105, no. 9, Article ID 093517, 6 pages, 2009.

[61] G. L. Eesley, "Generation of nonequilibrium electron and lattice temperatures in copper by picosecond laser pulses," Physical Review B, vol. 33, no. 4, pp. 2144-2151, 1986.

[62] P. M. Norris, A. P. Caffrey, R. J. Stevens, J. M. Klopf, J. T. McLeskey, and A. N. Smith, "Femtosecond pump-probe nondestructive examination of materials (invited)," Review of Scientific Instruments, vol. 74, no. 1, pp. 400-406, 2003.

[63] M. N. Touzelbaev, P. Zhou, R. Venkatasubramanian, and K. E. Goodson, "Thermal characterization of $\mathrm{Bi}_{2} \mathrm{Te}_{3} / \mathrm{Sb}_{2} \mathrm{Te}_{3}$ superlattices," Journal of Applied Physics, vol. 90, no. 2, pp. 763-767, 2001.

[64] P. M. Norris and P. E. Hopkins, "Examining interfacial diffuse phonon scattering through transient thermoreflectance measurements of thermal boundary conductance," Journal of Heat Transfer, vol. 131, no. 4, Article ID 043207, 11 pages, 2009.

[65] A. J. Schmidt, X. Chen, and G. Chen, "Pulse accumulation, radial heat conduction, and anisotropic thermal conductivity in pump-probe transient thermoreflectance," Review of Scientific Instruments, vol. 79, no. 11, Article ID 114902, 9 pages, 2008.

[66] P. E. Hopkins, J. R. Serrano, L. M. Phinney, S. P. Kearney, T. W. Grasser, and C. Thomas Harris, "Criteria for cross-plane dominated thermal transport in multilayer thin film systems during modulated laser heating," Journal of Heat Transfer, vol. 132, no. 8, Article ID 081302, 10 pages, 2010.

[67] P. E. Hopkins, B. Kaehr, L. M. Phinney et al., "Measuring the thermal conductivity of porous, transparent $\mathrm{SiO}_{2}$ films with time domain thermoreflectance," Journal of Heat Transfer, vol. 133, no. 6, Article ID 061601, 8 pages, 2011.

[68] Y. K. Koh, S. L. Singer, W. Kim et al., "Comparison of the $3 \omega$ method and time-domain thermoreflectance for measurements of the cross-plane thermal conductivity of epitaxial semiconductors," Journal of Applied Physics, vol. 105, no. 5, Article ID 054303, 7 pages, 2009.

[69] D. G. Cahill, "Analysis of heat flow in layered structures for time-domain thermoreflectance," Review of Scientific Instruments, vol. 75, no. 12, pp. 5119-5122, 2004.

[70] S. I. Anisimov, B. L. Kapeliovich, and T. L. Perelman, "Electron emission from metal surfaces exposed to ultrashort laser pulses," Soviet Physics, vol. 39, pp. 375-377, 1974.

[71] P. E. Hopkins, J. M. Klopf, and P. M. Norris, "Influence of interband transitions on electron-phonon coupling measurements in Ni films," Applied Optics, vol. 46, no. 11, pp. 2076-2083, 2007.

[72] P. E. Hopkins, L. M. Phinney, and J. R. Serrano, "Re-examining electron-fermi relaxation in gold films with a nonlinear thermoreflectance model," Journal of Heat Transfer, vol. 133, no. 4, Article ID 044505, 4 pages, 2011.

[73] P. E. Hopkins, J. L. Kassebaum, and P. M. Norris, "Effects of electron scattering at metal-nonmetal interfaces on electronphonon equilibration in gold films," Journal of Applied Physics, vol. 105, no. 2, Article ID 023710, 8 pages, 2009.

[74] P. E. Hopkins and P. M. Norris, "Substrate influence in electronphonon coupling measurements in thin Au films," Applied Surface Science, vol. 253, no. 15, pp. 6289-6294, 2007.
[75] P. E. Hopkins, "Influence of inter- and intraband transitions to electron temperature decay in noble metals after short-pulsed laser heating," Journal of Heat Transfer, vol. 132, no. 12, Article ID 122402, 6 pages, 2010.

[76] P. E. Hopkins, "Contributions of inter- and intraband excitations to electron heat capacity and electron-phonon coupling in noble metals," Journal of Heat Transfer, vol. 132, no. 1, Article ID 014504, 4 pages, 2010.

[77] J. Hohlfeld, S. S. Wellershoff, J. Güdde, U. Conrad, V. Jähnke, and E. Matthias, "Electron and lattice dynamics following optical excitation of metals," Chemical Physics, vol. 251, no. 1-3, pp. 237-258, 2000.

[78] T. Q. Qiu and C. L. Tien, "Heat transfer mechanisms during short-pulse laser heating of metals," Journal of Heat Transfer, vol. 115, no. 4, article 835, 7 pages, 1993.

[79] T. Q. Qiu and C. L. Tien, "Size effects on nonequilibrium laser heating of metal films," Journal of Heat Transfer, vol. 115, no. 4, article 842, 6 pages, 1993.

[80] A. Caffrey, P. Hopkins, J. Klopf, and P. Norris, "Thin film nonnoble transition metal thermophysical properties," Nanoscale and Microscale Thermophysical Engineering, vol. 9, no. 4, pp. 365-377, 2005.

[81] A. J. Schmidt, R. Cheaito, and M. Chiesa, "A frequency-domain thermoreflectance method for the characterization of thermal properties," Review of Scientific Instruments, vol. 80, no. 9, Article ID 094901, 6 pages, 2009.

[82] H. S. Carslaw and J. C. Jaeger, "Steady periodic temperature in composite slabs," in Conduction of Heat in Solids, pp. 109-112, Oxford University Press, New York, NY, USA, 2nd edition, 2003.

[83] A. Feldman, "Algorithm for solutions of the thermal diffusion equation in a stratified medium with a modulated heating source," High Temperatures, vol. 31, no. 3, pp. 293-296, 1999.

[84] A. Schmidt, M. Chiesa, X. Chen, and G. Chen, "An optical pump-probe technique for measuring the thermal conductivity of liquids," Review of Scientific Instruments, vol. 79, no. 6, Article ID 064902, 5 pages, 2008.

[85] D. G. Cahill and F. Watanabe, "Thermal conductivity of isotopically pure and Ge-doped Si epitaxial layers from 300 to $550 \mathrm{~K}$," Physical Review B, vol. 70, no. 23, Article ID 235322, 3 pages, 2004.

[86] F. Incropera and D. P. DeWitt, Fundamentals of Heat and Mass Transfer, John Wiley \& Sons, New York, NY, USA, 4th edition, 1996.

[87] D. R. Lide, CRC Handbook for Chemistry and Physics, Taylor \& Francis, Boca Raton, Fla, USA, 89th edition, 2008.

[88] Y. K. Koh and D. G. Cahill, "Frequency dependence of the thermal conductivity of semiconductor alloys," Physical Review $B$, vol. 76, no. 7, Article ID 075207, 5 pages, 2007.

[89] A. J. Minnich, J. A. Johnson, A. J. Schmidt et al., "Thermal conductivity spectroscopy technique to measure phonon mean free paths," Physical Review Letters, vol. 107, no. 9, Article ID 095901, 4 pages, 2011.

[90] R. J. Stevens, L. V. Zhigilei, and P. M. Norris, "Effects of temperature and disorder on thermal boundary conductance at solid-solid interfaces: nonequilibrium molecular dynamics simulations," International Journal of Heat and Mass Transfer, vol. 50, no. 19-20, pp. 3977-3989, 2007.

[91] J. C. Duda, T. S. English, E. S. Piekos, T. E. Beechem, T. W. Kenny, and P. E. Hopkins, "Bidirectionally tuning Kapitza conductance through the inclusion of substitutional impurities ," Journal of Applied Physics, vol. 112, no. 7, Article ID 073519, p. 5, 2012. 
[92] Z. Y. Ong and E. Pop, "Frequency and polarization dependence of thermal coupling between carbon nanotubes and $\mathrm{SiO}_{2}$," Journal of Applied Physics, vol. 108, no. 10, Article ID 103502, 8 pages, 2010.

[93] Z. Y. Ong and E. Pop, "Molecular dynamics simulation of thermal boundary conductance between carbon nanotubes and $\mathrm{SiO}_{2}$," Physical Review B, vol. 81, no. 15, Article ID 155408, 7 pages, 2010.

[94] E. S. Landry and A. J. H. McGaughey, "Effect of interfacial species mixing on phonon transport in semiconductor superlattices," Physical Review B, vol. 79, no. 7, Article ID 075316, 8 pages, 2009.

[95] E. S. Landry and A. J. H. McGaughey, "Thermal boundary resistance predictions from molecular dynamics simulations and theoretical calculations," Physical Review B, vol. 80, no. 16, Article ID 165304, 11 pages, 2009.

[96] Z. Huang, J. Y. Murthy, and T. S. Fisher, "Modeling of polarization-specific phonon transmission through interfaces," Journal of Heat Transfer, vol. 133, Article ID 114502, 3 pages, 2011.

[97] W. Zhang, T. S. Fisher, and N. Mingo, "Simulation of interfacial phonon transport in $\mathrm{Si}-\mathrm{Ge}$ heterostructures using an atomistic green's function method," Journal of Heat Transfer, vol. 129, no. 4, pp. 483-491, 2007.

[98] P. E. Hopkins, P. M. Norris, M. S. Tsegaye, and A. W. Ghosh, "Extracting phonon thermal conductance across atomic junctions: nonequilibrium green's function approach compared to semiclassical methods," Journal of Applied Physics, vol. 106, no. 6, Article ID 063503, 10 pages, 2009.

[99] N. Mingo, in Thermal Nanosystems and Nanomaterials, S. Volz, Ed., vol. 118 of Topic in Applied Physics, Springer, Berlin, Germany, 2009.

[100] W. Zhang, T. S. Fisher, and N. Mingo, "The atomistic Green's function method: an efficient simulation approach for nanoscale phonon transport," Numerical Heat Transfer B, vol. 51, no. 4, pp. 333-349, 2007.

[101] G. Laufer, Introduction to Optics and Lasers in Engineering, Cambridge University Press, Cambridge, UK, 1996.

[102] W. A. Little, "The transport of heat between dissimilar solids at low temperatures," Canadian Journal of Physics, vol. 37, no. 3, pp. 334-349, 1959.

[103] I. M. Khalatnikov and I. N. Adamenko, "Theory of the Kapitza temperature discontinuity at solid body-liquid helium boundary," Soviet Physics, vol. 36, p. 391, 1973.

[104] J. C. Duda, P. E. Hopkins, J. L. Smoyer et al., "On the assumption of detailed balance in prediction of diffusive transmission probability during interfacial transport," Nanoscale and Microscale Thermophysical Engineering, vol. 14, no. 1, pp. 21-33, 2010.

[105] E. T. Swartz and R. O. Pohl, "Thermal resistance at interfaces," Applied Physics Letters, vol. 51, no. 26, pp. 2200-2202, 1987.

[106] J. C. Duda, T. E. Beechem, J. L. Smoyer, P. M. Norris, and P. E. Hopkins, "Role of dispersion on phononic thermal boundary conductance," Journal of Applied Physics, vol. 108, no. 7, Article ID 073515, 10 pages, 2010.

[107] P. Reddy, K. Castelino, and A. Majumdar, "Diffuse mismatch model of thermal boundary conductance using exact phonon dispersion," Applied Physics Letters, vol. 87, no. 21, Article ID 211908, pp. 1-3, 2005.

[108] P. E. Hopkins, T. E. Beechem, J. C. Duda et al., "Influence of anisotropy on thermal boundary conductance at solid interfaces," Physical Review B, vol. 84, no. 12, Article ID 125408, 7 pages, 2011.
[109] R. S. Prasher, "Thermal boundary resistance and thermal conductivity of multiwalled carbon nanotubes," Physical Review $B$, vol. 77, no. 7, Article ID 075424, 11 pages, 2008.

[110] J. C. Duda, J. L. Smoyer, P. M. Norris, and P. E. Hopkins, "Extension of the diffuse mismatch model for thermal boundary conductance between isotropic and anisotropic materials," Applied Physics Letters, vol. 95, no. 3, Article ID 031912, 3 pages, 2009.

[111] W. G. Vincenti and C. H. Kruger, Introduction to Physical Gas Dynamics, Krieger Publishing Company, Malabar, Fla, USA, 2002.

[112] P. E. Hopkins, "Multiple phonon processes contributing to inelastic scattering during thermal boundary conductance at solid interfaces," Journal of Applied Physics, vol. 106, no. 1, Article ID 013528, 9 pages, 2009.

[113] P. E. Hopkins, J. C. Duda, and P. M. Norris, "Anharmonic phonon interactions at interfaces and contributions to thermal boundary conductance," Journal of Heat Transfer, vol. 133, Article ID 062401, 11 pages, 2011.

[114] C. Dames and G. Chen, "Theoretical phonon thermal conductivity of Si/Ge superlattice nanowires," Journal of Applied Physics, vol. 95, no. 2, pp. 682-693, 2004.

[115] G. Chen, "Diffusion-transmission interface condition for electron and phonon transport," Applied Physics Letters, vol. 82, no. 6, pp. 991-993, 2003.

[116] S. Simons, "On the thermal contact resistance between insulators," Journal of Physics C, vol. 7, no. 22, article 009, pp. 4048-4052, 1974.

[117] B. M. Clemens, G. L. Eesley, and C. A. Paddock, "Time-resolved thermal transport in compositionally modulated metal films," Physical Review B, vol. 37, no. 3, pp. 1085-1096, 1988.

[118] N. W. Ashcroft and N. D. Mermin, Solid State Physics, Saunders College, 1976.

[119] P. E. Hopkins, T. E. Beechem, J. C. Duda, J. L. Smoyer, and P. M. Norris, "Effects of subconduction band excitations on thermal conductance at metal-metal interfaces," Applied Physics Letters, vol. 96, no. 1, Article ID 011907, 3 pages, 2010.

[120] G. Wiedemann and R. Franz, "Ueber die warme-leitungsfahigkeit der Metalle," Annalen Der Physik, vol. 165, no. 4, pp. 978-531, 1853.

[121] G. D. Mahan and M. Bartkowiak, "Wiedemann-Franz law at boundaries," Applied Physics Letters, vol. 74, no. 7, pp. 953-954, 1999.

[122] P. E. Hopkins, R. J. Stevens, and P. M. Norris, "Influence of inelastic scattering at metal-dielectric interfaces," Journal of Heat Transfer, vol. 130, no. 2, Article ID 022401, 9 pages, 2008.

[123] T. Beechem, S. Graham, P. Hopkins, and P. Norris, "Role of interface disorder on thermal boundary conductance using a virtual crystal approach," Applied Physics Letters, vol. 90, no. 5, Article ID 054104, 2007.

[124] T. Beechem and P. E. Hopkins, "Predictions of thermal boundary conductance for systems of disordered solids and interfaces," Journal of Applied Physics, vol. 106, no. 12, Article ID 124301, 8 pages, 2009.

[125] B. Abeles, "Lattice thermal conductivity of disordered semiconductor alloys at high temperatures," Physical Review, vol. 131, no. 5, pp. 1906-1911, 1963.

[126] G. Gilat and R. M. Nicklow, "Normal vibrations in aluminum and derived thermodynamic properties," Physical Review, vol. 143, no. 2, pp. 487-494, 1966. 
[127] R. Weber, "Magnon-phonon coupling in metallic films," Physical Review, vol. 169, no. 2, pp. 451-456, 1968.

[128] G. P. Srivastava, The Physics of Phonons, Taylor \& Francis, New York, NY, USA, 1990.

[129] S. M. Lee, D. G. Cahill, and R. Venkatasubramanian, “Thermal conductivity of Si-Ge superlattices," Applied Physics Letters, vol. 70, no. 22, pp. 2957-2959, 1997.

[130] Z. Su, L. Huang, F. Liu et al., "Layer-by-layer thermal conductivities of the Group III nitride films in blue/green light emitting diodes," Applied Physics Letters, vol. 100, no. 20, Article ID 201106, 4 pages, 2012.

[131] D. G. Cahill, S. K. Watson, and R. O. Pohl, "Lower limit to the thermal conductivity of disordered crystals," Physical Review B, vol. 46, no. 10, pp. 6131-6140, 1992.

[132] A. Jallipalli, G. Balakrishnan, S. H. Huang et al., "Structural analysis of highly relaxed GaSb grown on GaAs substrates with periodic interfacial array of 90。 misfit dislocations," Nanoscale Research Letters, vol. 4, no. 12, pp. 1458-1462, 2009.

[133] S. H. Huang, G. Balakrishnan, A. Khoshakhlagh, A. Jallipalli, L. R. Dawson, and D. L. Huffaker, "Strain relief by periodic misfit arrays for low defect density GaSb on GaAs," Applied Physics Letters, vol. 88, no. 13, Article ID 131911, 3 pages, 2006.

[134] D. E. Gray, American Institute of Physics Handbook, McGraw Hill, New York, NY, USA, 3rd edition, 1972.

[135] M. K. Farr, J. G. Traylor, and S. K. Sinha, "Lattice dynamics of GaSb," Physical Review B, vol. 11, no. 4, pp. 1587-1594, 1975.

[136] K. Kunc and R. M. Martin, "Ab initio force constants of gaas: a new approach to calculation of phonons and dielectric properties," Physical Review Letters, vol. 48, no. 6, pp. 406-409, 1982.

[137] T. Beechem, J. C. Duda, P. E. Hopkins, and P. M. Norris, "Contribution of optical phonons to thermal boundary conductance," Applied Physics Letters, vol. 97, no. 6, Article ID 061907, 3 pages, 2010.

[138] J. C. Duda, T. S. English, E. S. Piekos, W. A. Soffa, L. V. Zhigilei, and P. E. Hopkins, "Implications of cross-species interactions on the temperature dependence of Kapitza conductance," Physical Review B, vol. 84, no. 19, Article ID 193301, 4 pages, 2011.

[139] M. Shen, W. J. Evans, D. Cahill, and P. Keblinski, "Bonding and pressure-tunable interfacial thermal conductance," Physical Review B, vol. 84, no. 19, Article ID 195432, 6 pages, 2011.

[140] L. Zhang, P. Keblinski, J. S. Wang, and B. Li, "Interfacial thermal transport in atomic junctions," Physical Review B, vol. 83, no. 6, Article ID 064303, 9 pages, 2011.

[141] L. Hu, L. Zhang, M. Hu, J. S. Wang, L. Baowen, and P. Keblinski, "Phonon interference at self-assembled monolayer interfaces: molecular dynamics simulations," vol. 81 , no. 23, Article ID 235427, 5 pages, 2010.

[142] M. Hu, P. Keblinski, and P. K. Schelling, "Kapitza conductance of silicon-amorphous polyethylene interfaces by molecular dynamics simulations," Physical Review B, vol. 79, no. 10, Article ID 104305, 7 pages, 2009.

[143] W. P. Hsieh, A. S. Lyons, E. Pop, P. Keblinski, and D. G. Cahill, "Pressure tuning of the thermal conductance of weak interfaces," Physical Review B, vol. 84, no. 18, Article ID 184107 , 5 pages, 2011.

[144] M. D. Losego, M. E. Grady, N. R. Sottos, D. G. Cahill, and P. V. Braun, "Effects of chemical bonding on heat transport across interfaces," Nature Materials, vol. 11, pp. 502-506, 2012.

[145] K. C. Collins and G. Chen, "Effects of surface chemistry on thermal conductance at aluminum-diamond interfaces,"
Applied Physics Letters, vol. 97, no. 8, Article ID 083102, 3 pages, 2010.

[146] C. Monachon and L. Weber, "Thermal boundary conductance of transition metals on diamond," Emerging Materials Research, vol. 1, pp. 89-98, 2012.

[147] M. Baraket, S. G. Walton, E. H. Lock, J. T. Robinson, and F. K. Perkins, "The functionalization of graphene using electronbeam generated plasmas," Applied Physics Letters, vol. 96, no. 23, Article ID 231501, 3 pages, 2010.

[148] E. H. Lock, M. Baraket, M. Laskoski et al., "High-quality uniform dry transfer of graphene to polymers," Nano Letters, vol. 12, pp. 102-107, 2012.

[149] R. J. Stoner, H. J. Maris, T. R. Anthony, and W. F. Banholzer, "Measurements of the Kapitza conductance between diamond and several metals," Physical Review Letters, vol. 68, no. 10, pp. 1563-1566, 1992.

[150] R. Nicklow, N. Wakabayashi, and H. G. Smith, "Lattice dynamics of pyrolytic graphite," Physical Review B, vol. 5, pp. 4951-4962, 1972.

[151] S. V. Kusminskiy, D. K. Campbell, and A. H. C. Neto, "Lenosky's energy and the phonon dispersion of graphene," Physical Review B, vol. 80, no. 3, Article ID 035401, 3 pages, 2009.

[152] W. Jang, Z. Chen, W. Bao, C. N. Lau, and C. Dames, “Thicknessdependent thermal conductivity of encased graphene and ultrathin graphite," Nano Letters, vol. 10, no. 10, pp. 3909-3913, 2010.

[153] Y. K. Koh, M. H. Bae, D. G. Cahill, and E. Pop, "Heat conduction across monolayer and few-layer graphenes," Nano Letters, vol. 10, no. 11, pp. 4363-4368, 2010.

[154] A. J. Schmidt, K. C. Collins, A. J. Minnich, and G. Chen, "Thermal conductance and phonon transmissivity of metalgraphite interfaces," Journal of Applied Physics, vol. 107, no. 10, Article ID 104907, 5 pages, 2010. 

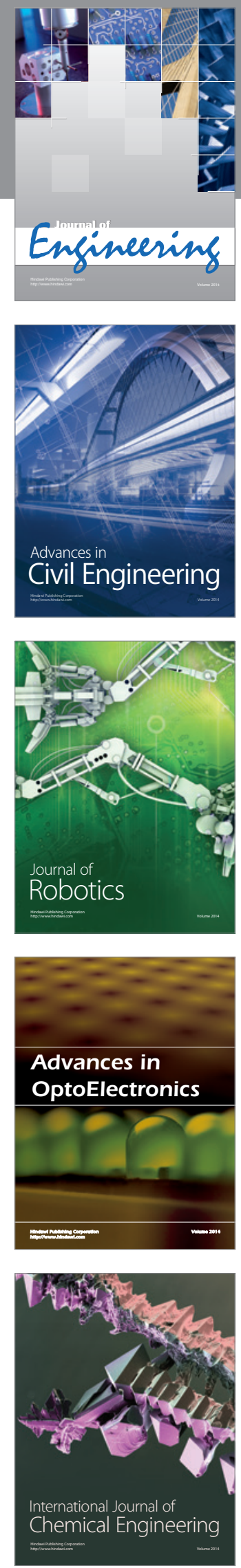

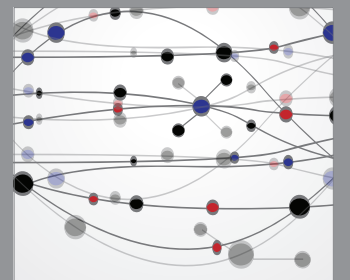

The Scientific World Journal
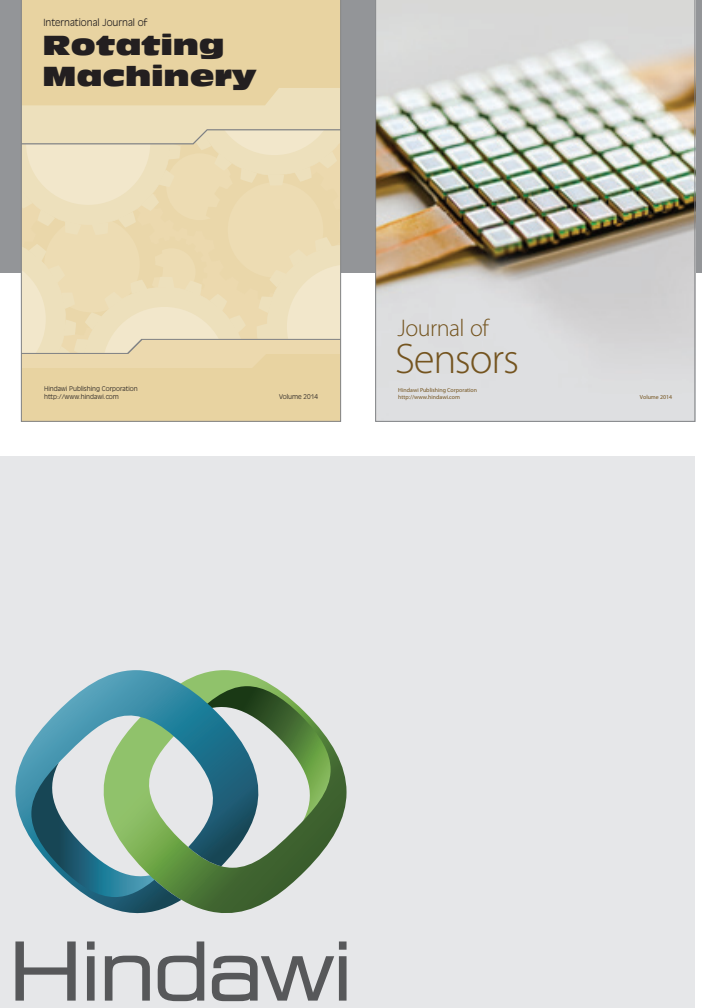

Submit your manuscripts at http://www.hindawi.com
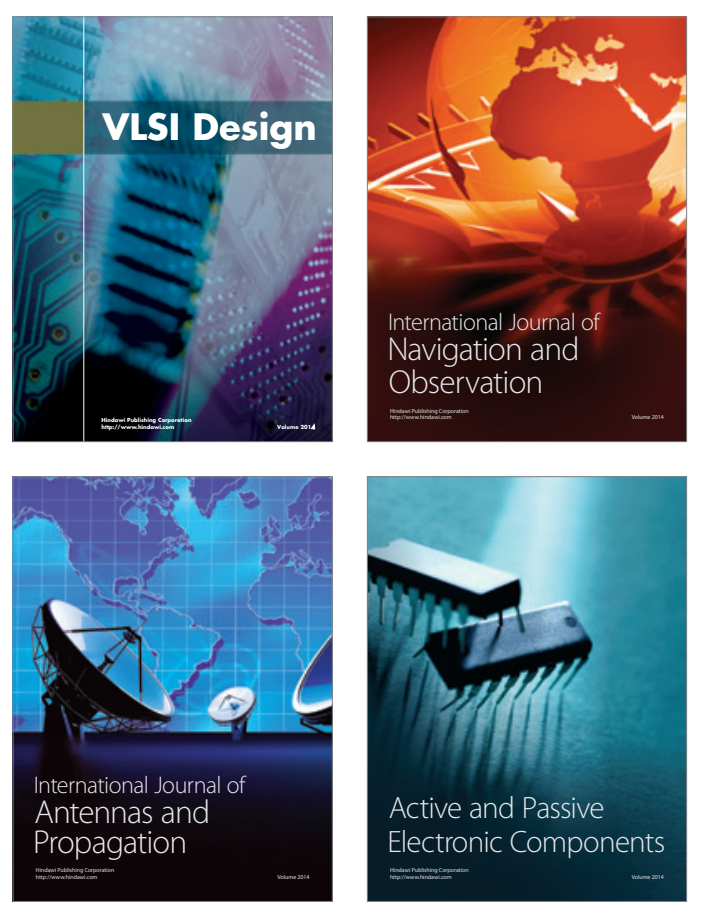
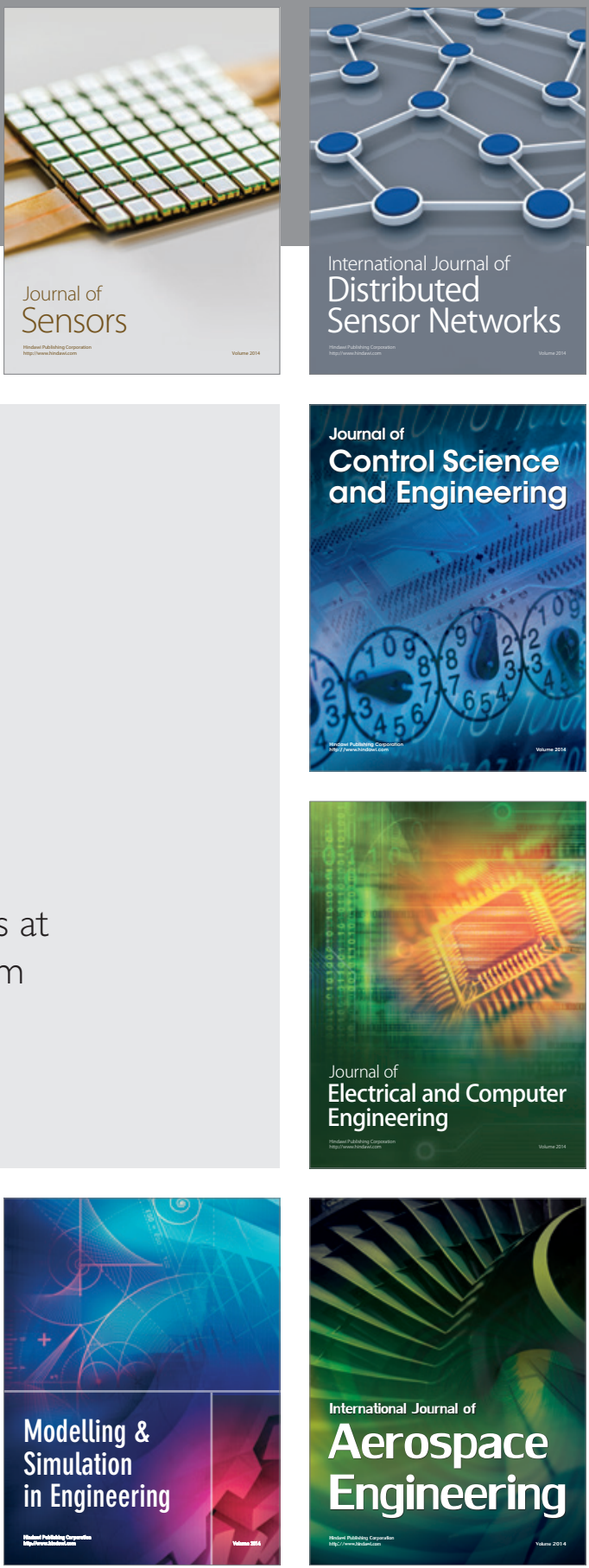

Journal of

Control Science

and Engineering
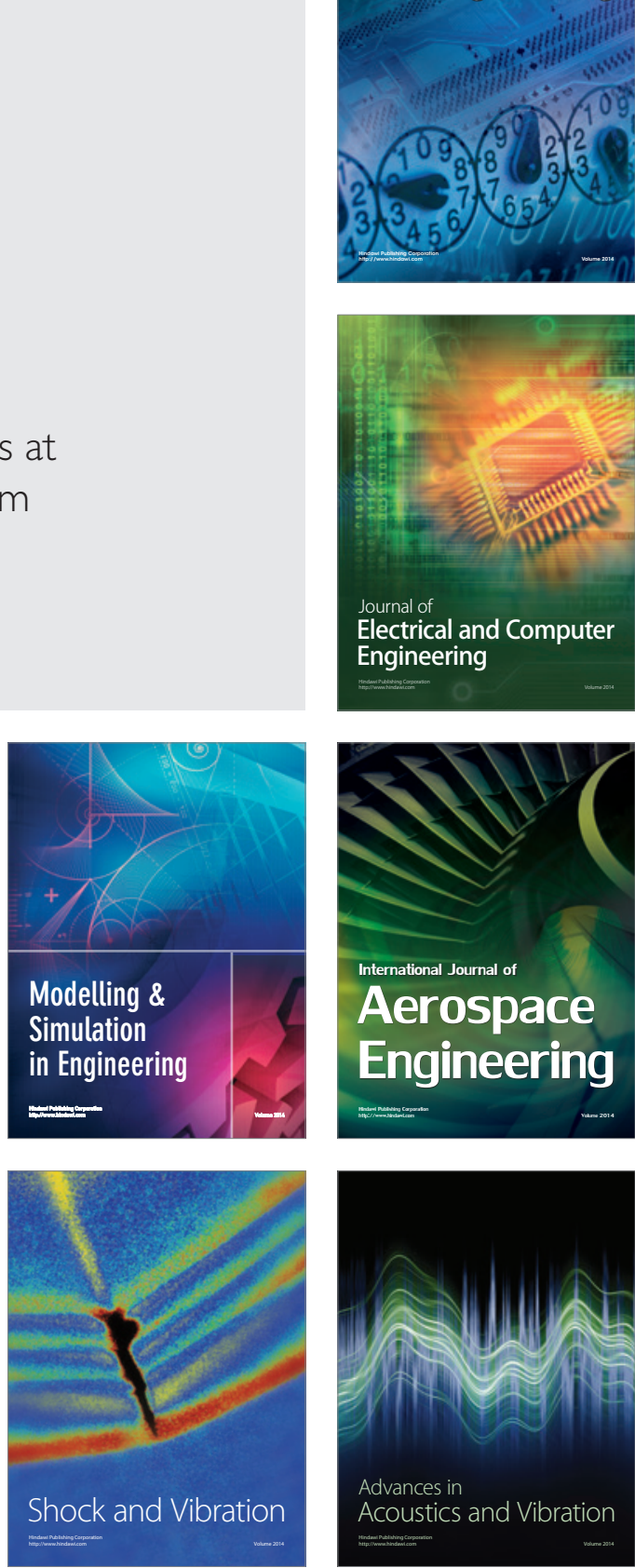